\title{
Maximum Norm Analysis of an Arbitrary Number of Nonmatching Grids Method for Nonlinears Elliptic PDES
}

\author{
Abida Harbi \\ Department of Mathematics, Laboratory of Applied Mathematics, Badji Mokhtar-Annaba University, P.O. Box 12, \\ El Hadjar, 23000 Annaba, Algeria
}

Correspondence should be addressed to Abida Harbi; a-harbi@hotmail.fr

Received 11 August 2013; Revised 7 October 2013; Accepted 9 October 2013

Academic Editor: Srinivasan Natesan

Copyright (C) 2013 Abida Harbi. This is an open access article distributed under the Creative Commons Attribution License, which permits unrestricted use, distribution, and reproduction in any medium, provided the original work is properly cited.

\begin{abstract}
We provide a maximum norm analysis of a finite element Schwarz alternating method for a nonlinear elliptic PDE on an arbitrary number of overlapping subdomains with nonmatching grids. We consider a domain which is the union of an arbitrary number $m$ of overlapping subdomains where each subdomain has its own independently generated grid. The $m$ meshes being mutually independent on the overlap regions, a triangle belonging to one triangulation does not necessarily belong to the other ones. Under the a Lipschitz assumption on the nonlinearity, we establish, on each subdomain, an optimal $L^{\infty}$ error estimate between the discrete Schwarz sequence and the exact solution of the PDE.
\end{abstract}

\section{Introduction}

The Schwarz alternating method can be used to solve elliptic boundary value problems on domains which consist of two or more overlapping subdomains. The solution is approximated by an infinite sequence of functions which result from solving a sequence of elliptic boundary value problems in each of the subdomains. The effectiveness of the Schwarz methods for various classes of nonlinear elliptic PDE problems has been demonstrated in many papers; see [1-4] and the references therein. Also the effectiveness of the Schwarz methods for these problems, especially those in fluid mechanics, has been demonstrated in many papers. See proceedings of the annual domain decomposition conference beginning with [5].

In $[6,7]$, an optimal convergence order is obtained for nonlinear elliptic PDE, in the context of two overlapping nonmatching grids, in the sense that each subdomain has its own independent discretization by finite element method. This kind of discretization is very interesting as it can be applied to solve many practical problems which cannot be handled by global discretizations. Discretizations are earning particular attention of computational experts and engineers as they allow the choice of different mesh sizes and different orders of approximate polynomials in different subdomains according to the different properties of the solution and different requirements of the practical problems. Quite a few works on maximum norm error analysis of overlapping nonmatching grids methods for elliptic problems are known in the literature; compare and confer, for example, [8-10].

This paper is a continuation of previous work [6], attempting to generalize the obtained result related to convergence order also in the context of nonmatching grids in domain which consists of a union of an arbitrary number of subdomains. It is proved that the error estimate remains true also for more than two subdomains.

The proof of the main result which consists of estimating the error in the maximum norm between the continuous solution of the problem and the discrete Schwarz iterates stands on a Lipschitz continuous dependency with respect to both the boundary condition and the source term for linear elliptic equations. The optimal convergence order is then derived making use of standard finite element $L^{\infty}$ error estimate for linear elliptic equations.

Now, we give an outline of the paper. In Section 2 we state the continuous alternating Schwarz sequences and define their respective finite element counterparts in the context of nonmatching overlapping grids. Section 3 is devoted to the $L^{\infty}$ error analysis of the method. 


\section{Preliminaries}

We begin by laying down some definitions and classical results related to linear elliptic equations.

2.1. Linear Elliptic Equations. Let $\Omega$ be a bounded polyhedral domain of $\mathbb{R}^{2}$ or $\mathbb{R}^{3}$ with sufficiently smooth boundary $\partial \Omega$. We consider the following: the bilinear form

$$
a(u, v)=\int_{\Omega}(\nabla u \cdot \nabla v) d x
$$

the linear form

$$
(f, v)=\int_{\Omega} f(x) \cdot v(x) d x
$$

the right hand side

$$
f \text {, a regular function; }
$$

the space

$$
V^{(g)}=\left\{v \in H^{1}(\Omega) \text { such that } v=g \text { on } \partial \Omega\right\},
$$

where $g$ is a regular function defined on $\partial \Omega$.

We consider the linear elliptic equation: Find $\zeta \in V^{(g)}$ such that

$$
a(\zeta, v)+c(\zeta, v)=(f, v), \quad \forall v \in V^{(g)}
$$

where $c \in \mathbb{R} c>0$, such that

$$
c \geq \beta>0 \text {. }
$$

Let $V_{h}$ be the space of finite elements consisting of continuous piecewise linear functions $v$ vanishing on $\partial \Omega$, and let $s, s=1,2, \ldots, m(h)$ be the basis function of $V_{h}$. The discrete counterpart of (5) consists of finding $\zeta_{h} \in V_{h}^{(g)}$ such that

$$
a\left(\zeta_{h}, v\right)+c\left(\zeta_{h}, v\right)=(f, v), \quad \forall v \in V_{h}^{(g)}
$$

where

$$
V_{h}^{(g)}=\left\{v \in V_{h}: v=\pi_{h} g \text { on } \partial \Omega\right\},
$$

and $\pi_{h}$ is an interpolation operator on $\partial \Omega$.

Theorem 1 (cf. [11]). Under the suitable regularity of the solution of problem (5), there exists a constant $C$ independent of $h$ such that

$$
\left\|\zeta-\zeta_{h}\right\|_{L^{\infty}(\Omega)} \leq C h^{2}|\log h|
$$

Lemma 2 (cf. [4]). Let $w \in H_{1}(\Omega) \cap C(\bar{\Omega})$ satisfy $a(w, \phi)+$ $c(w, \phi) \geq 0 \forall$ nonnegative $\phi \in H_{0}^{1}(\Omega)$, and $w \geq 0$ on $\partial \Omega$. Then $w \geq 0$ on $\bar{\Omega}$.

The proposition below establishes a Lipschitz continuous dependency of the solution with respect to the data.
Notation. Let $(f ; g) ;(\tilde{f}, \widetilde{g})$ be a pair of data and $\zeta=\sigma(f, g)$; $\widetilde{\zeta}=\sigma(\tilde{f}, \widetilde{g})$ the corresponding solutions to (5).

Proposition 3 (cf. [6]). Under conditions of the preceding lemma, one has:

$$
\|\zeta-\tilde{\zeta}\|_{L^{\infty}(\Omega)} \leq \max \left\{\frac{1}{\beta}\|f-\tilde{f}\|_{L^{\infty}(\Omega)},\|g-\tilde{g}\|_{L^{\infty}(\partial \Omega)}\right\} .
$$

Remark 4. Lemma 2 stays true in the discrete case.

Indeed, assume that the discrete maximum principle (d.m.p) holds; that is, the matrix resulting from the finite element discretization is an M-Matrix. Then one has the following.

Lemma 5. Let $w \in V_{h}$ satisfy $a(w, s)+c(w, s) \geq 0, s=$ $1,2, \ldots, m(h)$ and $w \geq 0$ on $\partial \Omega$. Then, $w \geq 0$ on $\bar{\Omega}$.

Proof. The proof is a direct consequence of the discrete maximum principle.

Let $(f, g) ;(\tilde{f}, \widetilde{g})$ be a pair of data and $\zeta_{h}=\sigma_{h}(f, g) ; \widetilde{\zeta}_{h}=$ $\sigma_{h}(\tilde{f}, \widetilde{g})$ the corresponding solutions to $(7)$.

Proposition 6 (cf. [6]). Let the d.m.p hold. Then, under conditions of Lemma 5, one has

$$
\left\|\zeta_{h}-\widetilde{\zeta}_{h}\right\|_{L^{\infty}(\Omega)} \leq \max \left\{\frac{1}{\beta}\|f-\tilde{f}\|_{L^{\infty}(\Omega)},\|g-\tilde{g}\|_{L^{\infty}(\partial \Omega)}\right\} .
$$

2.2. The Schwarz Alternating Methods for Nonlinear PDEs. Consider the nonlinear PDE:

$$
\begin{gathered}
-\Delta u+c u=f(u) \quad \text { in } \Omega, \\
u=0 \quad \text { on } \partial \Omega
\end{gathered}
$$

or in its weak form

$$
a(u, v)+c(u, v)=(f(u), v) \quad \forall v \in H_{0}^{1}(\Omega),
$$

where $f(\cdot)$ is a nondecreasing nonlinearity. We assume that $f(\cdot)$ is a Lipschitz continuous on $\mathbb{R}$, that is

$$
|f(x)-f(y)| \leq k|x-y|, \quad \forall x, y \in \mathbb{R}
$$

such that

$$
0<\frac{k}{\beta}<1,
$$

where $\beta$ is the constant defined in (6).

We suppose that the problem (12) has a subsolution $\underline{u}$ and a supersolution $\bar{u}$ which satisfy $0<\underline{u}<\bar{u}$. Define the sector of smooth functions as follows:

$$
A=\left\{u \in C^{2}(\Omega), \underline{u} \leq u \leq \bar{u} \text { on } \bar{\Omega}\right\} .
$$

Finally we assume that

$$
F(u)-F(v)<0, \quad v<u \in A,
$$


where

$$
F(u)=\frac{f(u)}{u} .
$$

With these assumptions cf. [12], the problem (12) has a unique solution in the sector $A$. We can easily see that (12) is equivalent to

$$
\begin{gathered}
-\Delta u=h(u) \quad \text { in } \Omega, \\
u=0 \quad \text { on } \partial \Omega,
\end{gathered}
$$

where $h(u)=f(u)-c u$, and we note that the functional $h(u)$ satisfies (17) and (18). We introduce the following comparison lemma.

Lemma 7 (cf. [3]). Suppose that $-\Delta u \geq h(u)$ and $-\Delta v \leq h(v)$ on $\Omega$ with both $u, v$ positive in $\Omega$ and $u \geq v \geq 0$ on $\partial \Omega$. Then $u \geq v$ on $\bar{\Omega}$.

We decompose $\Omega$ into $m$ overlapping smooth subdomains $\Omega_{i}$ such that

$$
\Omega=\bigcup_{i=1}^{m} \Omega_{i} .
$$

We denote by $\partial \Omega_{i}$ the boundary of $\Omega_{i}$ and the interior boundaries by

$$
\Gamma_{i}=\partial \Omega_{i} \cap \Omega .
$$

We assume that the intersection of $\bar{\Gamma}_{i}$ and $\bar{\Gamma}_{j}, i \neq j$ is empty. Let

$$
\begin{array}{r}
V_{i}^{\left(w_{j}\right)}=\left\{v \in C^{2}\left(\Omega_{i}\right) \text { such that } v=w_{j}\right. \\
\text { on } \left.\Gamma_{i}, v=0 \text { on } \partial \Omega_{i} \cap \partial \Omega\right\} .
\end{array}
$$

We associate problem (13) with the following system. Find $u_{i} \in \prod_{i=1}^{m} V_{i}^{\left(g_{i}\right)}$ solution of

$$
-\Delta u_{i}+c u_{i}=f\left(u_{i}\right) \quad \text { in } \Omega_{i},
$$

where

$$
u_{i}=\frac{u}{\Omega_{i}}, \quad i=1, \ldots, m
$$

and (cf. [13])

$$
g_{i}= \begin{cases}u_{i-1} & \text { on } \Gamma_{i} \cap \Omega_{i-1} \\ u_{i-2} & \text { on } \Gamma_{i} \cap\left(\Omega_{i-2}-\Omega_{i-1}\right) \\ \vdots & \text { on } \Gamma_{i} \cap\left(\Omega_{1}-\Omega_{i-1}-\cdots-\Omega_{1}\right) \\ u_{1} & \text { on } \Gamma_{i} \cap\left(\Omega_{m}-\bigcup_{j=1}^{i-1} \Omega_{j}\right) \\ u_{m-1} & \text { on } \Gamma_{i} \cap\left(\Omega_{m-1}-\bigcup_{j=1}^{i-1} \Omega_{j}-\Omega_{m}\right) \\ \vdots & \text { on } \Gamma_{i} \cap\left(\Omega_{i+1}-\bigcup_{j=1}^{i-1} \Omega_{j}-\bigcup_{j=i+2}^{m} \Omega_{j}\right) .\end{cases}
$$

In the sequel, we define a subsolution and a supersolution of (23)

Definition 8. A smooth function $\underline{u}_{i}$ is a subsolution of (23) if

$$
\begin{aligned}
& -\Delta \underline{u}_{i}+c \underline{u}_{i} \leq f\left(\underline{u}_{i}\right) \quad \text { in } \Omega_{i}, \\
& \underline{u}_{i}=\underline{g}_{i} \quad \text { on } \Gamma_{i}, \underline{u}_{i}=0 \text { on } \partial \Omega \cap \partial \Omega_{i},
\end{aligned}
$$

where

$$
\underline{g}_{i}= \begin{cases}\underline{u}_{i-1} & \text { on } \Gamma_{i} \cap \Omega_{i-1} \\ \underline{u}_{i-2} & \text { on } \Gamma_{i} \cap\left(\Omega_{i-2}-\Omega_{i-1}\right) \\ \vdots & \text { on } \Gamma_{i} \cap\left(\Omega_{1}-\Omega_{i-1}-\cdots-\Omega_{1}\right) \\ \underline{u}_{1} & \text { on } \Gamma_{i} \cap\left(\Omega_{m}-\bigcup_{j=1}^{i-1} \Omega_{j}\right) \\ \underline{u}_{m-1} & \text { on } \Gamma_{i} \cap\left(\Omega_{m-1}-\bigcup_{j=1}^{i-1} \Omega_{j}-\Omega_{m}\right) \\ \vdots & \text { on } \Gamma_{i} \cap\left(\Omega_{i+1}-\bigcup_{j=1}^{i-1} \Omega_{j}-\bigcup_{j=i+2}^{m} \Omega_{j}\right) .\end{cases}
$$

Similarly, a supersolution $\bar{u}_{i}$ of (23) satisfies

$$
\begin{gathered}
-\Delta \bar{u}_{i}+c \bar{u}_{i} \geq f\left(\bar{u}_{i}\right) \quad \text { in } \Omega_{i}, \\
u_{i}=\bar{g}_{i} \quad \text { on } \Gamma_{i}, \bar{u}_{i}=0 \text { on } \partial \Omega \cap \partial \Omega_{i} .
\end{gathered}
$$

2.3. The Continuous Schwarz Sequences. Let $u_{i}^{0}$ be an initialization data:

$$
u_{i}^{0}=\underline{u}_{i}, \quad i=1, \ldots, m .
$$

We define the alternating Schwarz sequences $\left(u_{i}^{n+1}\right)$ on $\Omega_{i}, \quad i=1, \ldots, m$, such that each term of each sequence $u_{i}^{n+1} \in$ $V^{\left(g_{i}^{n+1}\right)}, n \geq 0$ solves

$$
-\Delta u_{i}^{n+1}+c u_{i}^{n+1}=f\left(u_{i}^{n+1}\right) \quad \text { in } \Omega_{i},
$$

where (cf. [13])

$$
g_{i}^{n+1}= \begin{cases}u_{i-1}^{n+1} & \text { on } \Gamma_{i} \cap \Omega_{i-1} \\ u_{i-2}^{n+1} & \text { on } \Gamma_{i} \cap\left(\Omega_{i-2}-\Omega_{i-1}\right) \\ \vdots & \text { on } \Gamma_{i} \cap\left(\Omega_{1}-\Omega_{i-1}-\cdots-\Omega_{1}\right) \\ u_{1}^{n+1} & \text { on } \Gamma_{i} \cap\left(\Omega_{m}-\bigcup_{j=1}^{i-1} \Omega_{j}\right) \\ u_{m-1}^{n} & \text { on } \Gamma_{i} \cap\left(\Omega_{m-1}-\bigcup_{j=1}^{i-1} \Omega_{j}-\Omega_{m}\right) \\ \vdots & \text { on } \Gamma_{i} \cap\left(\Omega_{i+1}-\bigcup_{j=1}^{i-1} \Omega_{j}-\bigcup_{j=i+2}^{m} \Omega_{j}\right) .\end{cases}
$$


Theorem 9. The Schwarz sequences $\left(u_{i}^{n+1}\right), i=1, \ldots, m$ converge to the solution of (23).

Proof. (1) We first show by induction, that each term of the Schwarz sequences is well defined in $A$. Indeed, for $n=0$, we begin by subdomain one, since $u_{1}^{1}, \bar{u}_{1}$ and $\underline{u}_{1}$ satisfy

$$
-\Delta u_{1}^{1}+c u_{1}^{1}=f\left(u_{1}^{1}\right) \text { in } \Omega_{1}, \quad u_{1}^{1}=0 \text { on } \partial \Omega_{1} \cap \partial \Omega,
$$

$$
\begin{gathered}
-\Delta \bar{u}_{1}+c \bar{u}_{1} \geq f\left(\bar{u}_{1}\right) \quad \text { in } \Omega_{1}, \\
\bar{u}_{1}=0 \quad \text { on } \partial \Omega_{1} \cap \partial \Omega, \\
-\Delta \underline{u}_{1}+c \underline{u}_{1} \leq f\left(\underline{u}_{1}\right) \quad \text { in } \Omega_{1}, \\
\underline{u}_{1}=0 \quad \text { on } \partial \Omega_{1} \cap \partial \Omega
\end{gathered}
$$

respectively with

$$
u_{1}^{1}=g_{1}^{1}=\underline{g}_{1} \leq \bar{g}_{1} \quad \text { on } \Gamma_{1}
$$

Lemma 7 implies that

$$
\underline{u}_{1} \leq u_{1}^{1} \leq \bar{u}_{1} \quad \text { in } \bar{\Omega}_{1} .
$$

In subdomain 2 , the condition of the interior boundary $\Gamma_{2}$ is given by

$$
g_{2}^{1}= \begin{cases}u_{1}^{1} & \text { on } \Gamma_{2} \cap \Omega_{1} \\ u_{m}^{0} & \text { on } \Gamma_{2} \cap\left(\Omega_{m}-\Omega_{1}\right) \\ u_{m-1}^{0} & \text { on } \Gamma_{2} \cap\left(\Omega_{m-1}-\Omega_{1}-\Omega_{m}\right) \\ \vdots & \\ u_{3}^{n} & \text { on } \Gamma_{2} \cap\left(\Omega_{3}-\Omega_{1}-\bigcup_{j=4}^{m} \Omega_{j}\right) .\end{cases}
$$

so (37) implies that

$$
\underline{g}_{2} \leq g_{2}^{1} \leq \bar{g}_{2} \quad \text { on } \Gamma_{2}
$$

On the other hand $u_{2}^{1}, \bar{u}_{2}$, and $\underline{u}_{2}$ satisfy, respectively,

$$
\begin{gathered}
-\Delta u_{2}^{1}+c u_{2}^{1}=f\left(u_{2}^{1}\right) \quad \text { in } \Omega_{2}, \\
u_{2}^{1}=0 \text { on } \partial \Omega_{2} \cap \partial \Omega, \\
-\Delta \bar{u}_{2}+c \bar{u}_{2} \geq f\left(\bar{u}_{2}\right) \quad \text { in } \Omega_{2}, \\
\bar{u}_{2}=0 \text { on } \partial \Omega_{2} \cap \partial \Omega, \\
-\Delta \underline{u}_{2}+c \underline{u}_{2} \leq f\left(\underline{u}_{2}\right) \quad \text { in } \Omega_{2}, \\
\underline{u}_{2}=\text { on } \partial \Omega_{2} \cap \partial \Omega .
\end{gathered}
$$

Then Lemma 7 implies that

$$
\underline{u}_{2} \leq u_{2}^{1} \leq \bar{u}_{2} \quad \text { in } \bar{\Omega}_{2} .
$$

The same idea is used sequentially in the remainder of the proof related to the rest of subdomains $\Omega_{i}, 3 \leq i \leq m$. Indeed we obtain for $i=1, \ldots, m$ and for $n=0$

$$
\underline{u}_{i} \leq u_{i}^{1} \leq \bar{u}_{i} \quad \text { in } \bar{\Omega}_{i} .
$$

Now, let us assume that

$$
\underline{u}_{i} \leq u_{i}^{n} \leq \bar{u}_{i} \quad \text { in } \bar{\Omega}_{i}, i=1, \ldots, m
$$

and prove that

$$
\underline{u}_{i} \leq u_{i}^{n+1} \leq \bar{u}_{i} \quad \text { in } \bar{\Omega}_{i}, i=1, \ldots, m \text {. }
$$

Indeed, in subdomain 1 (43) implies that

$$
\underline{g}_{1} \leq g_{1}^{n+1} \leq \bar{g}_{1} \quad \text { on } \Gamma_{1}
$$

Since

$$
\begin{gathered}
-\Delta u_{1}^{n+1}+c u_{1}^{n+1}=f\left(u_{1}^{n+1}\right) \quad \text { in } \Omega_{1}, \\
u_{1}^{n+1}=0 \quad \text { on } \partial \Omega_{1} \cap \partial \Omega, \\
-\Delta \bar{u}_{1}+c \bar{u}_{1} \geq f\left(\bar{u}_{1}\right) \quad \text { in } \Omega_{1}, \\
\bar{u}_{1}=0 \quad \text { on } \partial \Omega_{1} \cap \partial \Omega, \\
-\Delta \underline{u}_{1}+c \underline{u}_{1} \leq f\left(\underline{u}_{1}\right) \quad \text { in } \Omega_{1}, \\
\underline{u}_{1}=0 \quad \text { on } \partial \Omega_{1} \cap \partial \Omega,
\end{gathered}
$$

then Lemma 7 implies that

$$
\underline{u}_{1} \leq u_{1}^{n+1} \leq \bar{u}_{1} \quad \text { in } \bar{\Omega}_{1} .
$$

Using (47) and (43), we can write

$$
\underline{g}_{2} \leq g_{2}^{n+1} \leq \bar{g}_{2} \quad \text { on } \Gamma_{2} \text {. }
$$

We can easily obtain

$$
\underline{u}_{2} \leq u_{2}^{n+1} \leq \bar{u}_{2} \quad \text { in } \bar{\Omega}_{2}
$$

using (48) and by adopting the same approach used in subdomain one. The result related to rest of subdomains $\Omega_{i}$, $3 \leq i \leq m$, is obtained sequentially by similar way; that is

$$
\underline{u}_{i} \leq u_{i}^{n+1} \leq \bar{u}_{i} \quad \text { in } \bar{\Omega}_{i}, i=1, \ldots, m, n \geq 0 .
$$

(2) We demonstrate, by induction, that the Schwarz sequences are nondecreasing. Indeed for $n=0$, we have demonstrated in (50) that, for $i=1, \ldots, m$,

$$
u_{i}^{0} \leq u_{i}^{1} \quad \text { in } \bar{\Omega}_{i}
$$

Now, let us assume that

$$
u_{i}^{n-1} \leq u_{i}^{n} \quad \text { in } \bar{\Omega}_{i}, i=1, \ldots, m
$$

and prove that

$$
u_{i}^{n} \leq u_{i}^{n+1} \quad \text { in } \bar{\Omega}_{i}, i=1, \ldots, m .
$$

Begining by subdomain one, (52) implies that

$$
g_{1}^{n} \leq g_{1}^{n+1} \quad \text { on } \Gamma_{1} \text {. }
$$


Making use of (54),

$$
\begin{gathered}
-\Delta u_{1}^{n+1}+c u_{1}^{n+1}=f\left(u_{1}^{n+1}\right) \quad \text { in } \Omega_{1}, \\
u_{1}^{n+1}=0 \quad \text { on } \partial \Omega_{1} \cap \partial \Omega, \\
-\Delta u_{1}^{n}+c u_{1}^{n}=f\left(u_{1}^{n}\right) \quad \text { in } \Omega_{1}, \\
u_{1}^{n}=0 \quad \text { on } \partial \Omega_{1} \cap \partial \Omega .
\end{gathered}
$$

We get by using Lemma 7

$$
u_{1}^{n} \leq u_{1}^{n+1} \quad \text { in } \bar{\Omega}_{1} .
$$

On the other hand, (52) and (56) imply

$$
g_{2}^{n} \leq g_{2}^{n+1} \quad \text { on } \Gamma_{2}
$$

then making use of (57),

$$
\begin{gathered}
-\Delta u_{2}^{n+1}+c u_{2}^{n+1}=f\left(u_{2}^{n+1}\right) \quad \text { in } \Omega_{2}, \\
u_{2}^{n+1}=0 \quad \text { on } \partial \Omega_{2} \cap \partial \Omega, \\
-\Delta u_{2}^{n}+c u_{2}^{n}=f\left(u_{2}^{n}\right) \quad \text { in } \Omega_{2}, \\
u_{2}^{n}=0 \quad \text { on } \partial \Omega_{2} \cap \partial \Omega .
\end{gathered}
$$

We get by using Lemma 7 the following result related to the second subdomain:

$$
u_{2}^{n} \leq u_{2}^{n+1} \text { in } \bar{\Omega}_{2} .
$$

The same idea is sequentially applied to the rest of subdomains. Finally, we obtain

$$
u_{i}^{n} \leq u_{i}^{n+1} \quad \text { in } \bar{\Omega}_{i}, i=1, \ldots, m, n \geq 0 .
$$

(3) The sequences $\left(u_{i}^{n}\right)$ are bounded and nondecreasing, so they are monotone converging pointwise to $u_{i}, i=$ $1, \ldots, m$. By using an elliptic regularity argument, we can see that functions $u_{i}$ satisfy the same PDE on $\Omega_{i} \mathrm{cf}$. $[3,12]$.

2.4. The Discretization. For $i=1, \ldots, m$, let $\tau^{h_{i}}$ be a standard regular and quasiuniform finite element triangulation in $\Omega_{i}$; $h_{i}$ being the meshsize. The $m$ meshes is mutually independent on $\Omega_{i} \cap \Omega_{j}, i, j=1, \ldots, m$, where $i \neq j$; that is a triangle belonging to one triangulation does not necessarily belong to the other ones.

We consider the following discrete spaces:

$$
V_{h_{i}}=\left\{v \in C\left(\bar{\Omega}_{i}\right) \cap H^{1}\left(\Omega_{i}\right) \text { such that } \frac{v}{K} \in P_{1} \quad \forall K \in \tau^{h_{i}}\right\} \text {, }
$$

and for every $w \in C\left(\bar{\Gamma}_{i}\right)$, we set

$$
V_{h_{i}}^{(w)}=\left\{v \in V_{h_{i}}: v=0 \text { on } \partial \Omega_{i} \cap \partial \Omega ; \quad v=\pi_{h_{i}}(w) \text { on } \Gamma_{i}\right\},
$$

where $\pi_{h_{i}}$ denotes the interpolation operator on $\Gamma_{i}$.
The Discrete Maximum Principle ( $c f .[14,15])$. We assume that the respective matrices resulting from the discretiztions of problems (30) are M-matrices.

Note that as the meshes $h_{i}$ are independent over the overlapping subdomains, it is impossible to formulate a global approximate problem which would be the direct discrete counterpart of problem (13).

2.5. The Discrete Schwarz Sequences. Now, we define the discrete counterparts of the continuous Schwarz sequences defined in (30). Indeed, let $u_{h}^{0}$ be the discrete analog of $u_{0}$, defined in (29) and we define for $i=1, \ldots, m$, the discrete sequences $\left(u_{h_{i}}^{n+1}\right)$ on $\Omega_{i}$ such that $u_{h_{i}}^{n+1} \in V_{h_{i}}^{\left(g_{h_{i}}^{n+1}\right)}$ solves

$$
\begin{array}{r}
a_{i}\left(u_{h_{i}}^{n+1}, v\right)+c\left(u_{h_{i}}^{n+1}, v\right)=\left(f\left(u_{h_{i}}^{n+1}\right), v\right), \\
\forall v \in V_{h_{i}}^{\left(g_{h_{i}}^{n+1}\right)} ; n \geq 0,
\end{array}
$$

where

$$
\begin{aligned}
& a_{i}(u, v)=\int_{\Omega_{i}}(\nabla u \cdot \nabla v) d x \text {, } \\
& g_{h_{i}}^{n+1}= \begin{cases}u_{h_{i-1}}^{n+1} & \text { on } \Gamma_{i} \cap \Omega_{i-1} \\
u_{h_{i-2}}^{n+1} & \text { on } \Gamma_{i} \cap\left(\Omega_{i-2}-\Omega_{i-1}\right) \\
\vdots & \text { on } \Gamma_{i} \cap\left(\Omega_{1}-\Omega_{i-1}-\cdots-\Omega_{1}\right) \\
u_{h_{1}}^{n+1} & \text { on } \Gamma_{i} \cap\left(\Omega_{m}-\bigcup_{j=1}^{i-1} \Omega_{j}\right) \\
u_{h_{m-1}}^{n} & \text { on } \Gamma_{i} \cap\left(\Omega_{m-1}-\bigcup_{j=1}^{i-1} \Omega_{j}-\Omega_{m}\right) \\
\vdots & \text { on } \Gamma_{i} \cap\left(\Omega_{i+1}-\bigcup_{j=1}^{i-1} \Omega_{j}-\bigcup_{j=i+1}^{m} \Omega_{j}\right) .\end{cases}
\end{aligned}
$$

Notation. From now on, we will adopt the following notations $i=1, \ldots, m$ :

$$
\begin{gathered}
|\cdot|_{i}=\|\cdot\|_{L^{\infty}\left(\Gamma_{i}\right)} \quad\|\cdot\|_{i}=\|\cdot\|_{L^{\infty}\left(\Omega_{i}\right)}, \\
\pi_{h_{i}}=\pi_{h} .
\end{gathered}
$$

\section{3. $L^{\infty}$ Error Analysis}

3.1. The Auxiliary Schwarz Problems. This section is devoted to the proof of the main result of the present paper. To that end, we begin by introducing $m$ discrete auxiliary problems.

We define the following problems; for $i=1, \ldots, m, w_{h_{i}} \in$ $V_{h_{i}}^{\left(g_{i}\right)}$ solves

$$
a_{i}\left(w_{h_{i}}, v\right)+c\left(w_{h_{i}}, v\right)=\left(f\left(u_{i}\right), v\right) \quad \forall v \in V_{h_{i}}^{\left(g_{i}\right)} .
$$

It is then clear that $w_{h_{i}}$ is the finite element approximation of $u_{i}$ solutions of (23). Therefore, making use of standard 
maximum norm estimates for linear elliptic problems, we have

$$
\left\|u_{i}-w_{h_{i}}\right\|_{i} \leq C h^{2}|\log h|
$$

where $C$ is a constant independent of $h$.

\subsubsection{The Main Results}

Theorem 10. Let $\rho=k / \beta<1$. Then, there exists a constant $C$ independent of both $h$ and $n$ such that

$$
\left\|u_{i}-u_{h_{i}}^{n+1}\right\|_{i} \leq \frac{1}{1-\rho} C h^{2}|\log h| ; \quad n \geq 0 ; i=1, \ldots, m .
$$

Proof. The proof of (68) will be carried by induction and is decomposed in three principal parts where each part is devoted to one of the following situations: situation (A), situation (B), and situation (C), respectively. The three situations are defined by

$$
\begin{gathered}
\text { (A) } \max _{1 \leq i \leq m}\left\|u_{i}-u_{h_{i}}^{0}\right\|_{i}<C h^{2}|\log h|, \\
\text { (B) } C h^{2}|\log h|<\left\|u_{i}-u_{h_{i}}^{0}\right\|_{i}, \quad i=1, \ldots, m,
\end{gathered}
$$

and in the last situation we assume that a nonempty subset $J$ of the set $M=\{1, \ldots, m\}$ exists such that

(C) $\forall i \in M-J ;\left\|u_{i}-u_{h_{i}}^{0}\right\|_{i}<C h^{2}|\log h|<\left\|u_{j}-u_{h_{j}}^{0}\right\|_{j}$, $\forall j \in J$.

To this end, we apply Proposition 6 and Theorem 1 for each subdomain $\Omega_{i}$ as follows:

$$
\begin{aligned}
\left\|u_{i}-u_{h_{i}}^{n+1}\right\|_{i} \leq & \left\|u_{i}-w_{h_{i}}\right\|_{i}+\left\|w_{h_{i}}-u_{h_{i}}^{n+1}\right\|_{i} \\
\leq & C h^{2}|\log h|+\left\|w_{h_{i}}-u_{h_{i}}^{n+1}\right\|_{i} \\
\leq & C h^{2}|\log h| \\
& +\max \left\{\left(\frac{1}{\beta}\right)\left\|f\left(u_{i}\right)-f\left(u_{h_{i}}^{n+1}\right)\right\|_{i},\left|g_{i}-g_{h_{i}}^{n+1}\right|_{i}\right\} \\
\leq & C h^{2}|\log h| \\
& +\max \left\{\left(\frac{1}{\beta}\right)\left\|f\left(u_{i}\right)-f\left(u_{h_{i}}^{n+1}\right)\right\|_{i},\right. \\
& \left.\max _{j<i}\left\|u_{j}-u_{h_{j}}^{n+1}\right\|_{j}, \max _{i<j}\left\|_{u_{j}}-u_{h_{j}}^{n}\right\|_{j}\right\} .
\end{aligned}
$$

The last inequality implies that

$$
\begin{aligned}
\left\|u_{i}-u_{h_{i}}^{n+1}\right\|_{i} \leq & C h^{2}|\log h| \\
& +\max \left\{\rho\left\|u_{i}-u_{h_{i}}^{n+1}\right\|_{i}, \max _{j<i}\left\|u_{j}-u_{h_{j}}^{n+1}\right\|_{j}\right.
\end{aligned}
$$

$$
\left.\max _{i<j}\left\|u_{j}-u_{h_{j}}^{n}\right\|_{j}\right\} \text {. }
$$

The interval ]0,1[ which $\rho$ belongs to is divided into two subintervals as follows:

$$
] 0,1[=] 0, \frac{1}{2}\left[\cup \left[\frac{1}{2}, 1[\right.\right.
$$

and in each situation (A), (B), or (C) we deal with each subinterval separately.

Part 1. We consider situation (A). We begin the proof by the first subinterval that is $\rho \in] 0,1 / 2[$, so

$$
\frac{\rho}{1-\rho}<1
$$

For $n=0$, applying (73) to subdomain one, we get

$$
\begin{aligned}
\left\|u_{1}-u_{h_{1}}^{1}\right\|_{1} \leq & C h^{2}|\log h| \\
& +\max \left\{\rho\left\|u_{1}-u_{h_{1}}^{1}\right\|_{1}, \max _{1<j \leq m}\left\|u_{j}-u_{h_{j}}^{0}\right\|_{j}\right\} .
\end{aligned}
$$

We have to distinguish between two cases:

$$
\text { (1) } \max \left\{\rho\left\|u_{1}-u_{h_{1}}^{1}\right\|_{1}, \max _{1<j \leq m}\left\|u_{j}-u_{h_{j}}^{0}\right\|_{j}\right\}=\rho\left\|u_{1}-u_{h_{1}}^{1}\right\|_{1}
$$

or

$$
\begin{gathered}
\text { (2) } \max \left\{\rho\left\|u_{1}-u_{h_{1}}^{1}\right\|_{1}, \max _{1<j \leq m}\left\|u_{j}-u_{h_{j}}^{0}\right\|_{j}\right\} \\
=\max _{1<j \leq m}\left\|u_{j}-u_{h_{j}}^{0}\right\|_{j}
\end{gathered}
$$

Case 1 implies that

$$
\begin{gathered}
\left\|u_{1}-u_{h_{1}}^{1}\right\|_{1} \leq C h^{2}|\log h|+\rho\left\|u_{1}-u_{h_{1}}^{1}\right\|_{1}, \\
\max _{1<j \leq m}\left\|u_{j}-u_{h_{j}}^{0}\right\|_{j} \leq \rho\left\|u_{1}-u_{h_{1}}^{1}\right\|_{1} .
\end{gathered}
$$

Then,

$$
\begin{aligned}
\left\|u_{1}-u_{h_{1}}^{1}\right\|_{1} \leq & \frac{1}{1-\rho} C h^{2}|\log h|, \\
\max _{1<j \leq m}\left\|u_{j}-u_{h_{j}}^{0}\right\|_{j} & \leq \rho\left\|u_{1}-u_{h_{1}}^{1}\right\|_{1} \\
& \leq \frac{\rho}{1-\rho} C h^{2}|\log h| \\
& <C h^{2}|\log h| .
\end{aligned}
$$


Case 2 implies that

$$
\begin{gathered}
\left\|u_{1}-u_{h_{1}}^{1}\right\|_{1} \leq C h^{2}|\log h|+\max _{1<j \leq m}\left\|u_{j}-u_{h_{j}}^{0}\right\|_{j}, \\
\rho\left\|u_{1}-u_{h_{1}}^{1}\right\|_{1} \leq \max _{1<j \leq m}\left\|u_{j}-u_{h_{j}}^{0}\right\|_{j} .
\end{gathered}
$$

By multiplying (81) by $\rho$, we get

$$
\begin{gathered}
\rho\left\|u_{1}-u_{h_{1}}^{1}\right\|_{1} \leq \rho C h^{2}|\log h|+\rho \max _{1<j \leq m}\left\|u_{j}-u_{h_{j}}^{0}\right\|_{j}, \\
\rho\left\|u_{1}-u_{h_{1}}^{1}\right\|_{1} \leq \max _{1<j \leq m}\left\|u_{j}-u_{h_{j}}^{0}\right\|_{j} .
\end{gathered}
$$

So $\rho\left\|u_{1}-u_{h_{1}}^{1}\right\|_{1}$ is bounded by both $\rho C h^{2}|\log h|+\rho \max _{1<j \leq m}$ $\left\|u_{j}-u_{h_{j}}^{0}\right\|_{j}$ and $\max _{1<j \leq m}\left\|u_{j}-u_{h_{j}}^{0}\right\|_{j}$, then

(a) $\max _{1<j \leq m}\left\|u_{j}-u_{h_{j}}^{0}\right\|_{j}<\rho C h^{2}|\log h|+\rho \max _{1<j \leq m}\left\|u_{j}-u_{h_{j}}^{0}\right\|_{j}$

or

(b) $\rho C h^{2}|\log h|+\rho \max _{1<j \leq m}\left\|u_{j}-u_{h_{j}}^{0}\right\|_{j}<\max _{1<j \leq m}\left\|u_{j}-u_{h_{j}}^{0}\right\|_{j}$.

That is,

$$
\max _{1<j \leq m}\left\|u_{j}-u_{h_{j}}^{0}\right\|_{j}<\frac{\rho}{1-\rho} C h^{2}|\log h|
$$

or

$$
\frac{\rho}{1-\rho} C h^{2}|\log h|<\max _{1<j \leq m}\left\|u_{j}-u_{h_{j}}^{0}\right\|_{j}
$$

which implies that

$$
\max _{1<j \leq m}\left\|u_{j}-u_{h_{j}}^{0}\right\|_{j}<\frac{\rho}{1-\rho} C h^{2}|\log h|<C h^{2}|\log h|,
$$

or

$$
\frac{\rho}{1-\rho} C h^{2}|\log h|<\max _{1<j \leq m}\left\|u_{j}-u_{h_{j}}^{0}\right\|_{j}<C h^{2}|\log h| .
$$

It is clear that both cases (a) and (b) are true because both coincide with (69). So for either of them there is a contradiction, and thus case 2 is impossible or case 2 is possible only if

$$
\max _{1<j \leq m}\left\|u_{j}-u_{h_{j}}^{0}\right\|_{j}=\frac{\rho}{1-\rho} C h^{2}|\log h| .
$$

Then case 2 implies that

$$
\begin{aligned}
\left\|u_{1}-u_{h_{1}}^{1}\right\|_{1} & \leq C h^{2}|\log h|+\max _{1<j \leq m}\left\|u_{j}-u_{h_{j}}^{0}\right\|_{j} \\
& =C h^{2}|\log h|+\frac{\rho}{1-\rho} C h^{2}|\log h| \\
& =\frac{1}{1-\rho} C h^{2}|\log h| .
\end{aligned}
$$

Hence in both cases 1 and 2, we obtain

$$
\left\|u_{1}-u_{h_{1}}^{1}\right\|_{1} \leq \frac{1}{1-\rho} C h^{2}|\log h|
$$

Similarly applying (73) to the second subdomain, we get

$$
\begin{aligned}
&\left\|u_{2}-u_{h_{2}}^{1}\right\|_{2} \leq C h^{2}|\log h| \\
&+\max \left\{\rho\left\|u_{2}-u_{h_{2}}^{1}\right\|_{2},\left\|u_{1}-u_{h_{1}}^{1}\right\|_{1},\right. \\
&\left.\max _{2<j \leq m}\left\|u_{j}-u_{h_{j}}^{0}\right\|_{j}\right\} .
\end{aligned}
$$

We have to distinguish the three following cases

$$
\begin{aligned}
& \text { (1) } \max \left\{\rho\left\|u_{2}-u_{h_{2}}^{1}\right\|_{2},\left\|u_{1}-u_{h_{1}}^{1}\right\|_{1}, \max _{2<j \leq m}\left\|u_{j}-u_{h_{j}}^{0}\right\|_{j}\right\} \\
& =\rho\left\|u_{2}-u_{h_{2}}^{1}\right\|_{2}
\end{aligned}
$$

or

$$
\begin{aligned}
& \text { (2) } \max \left\{\rho\left\|u_{2}-u_{h_{2}}^{1}\right\|_{2},\left\|u_{1}-u_{h_{1}}^{1}\right\|_{1}, \max _{2<j \leq m}\left\|u_{j}-u_{h_{j}}^{0}\right\|_{j}\right\} \\
& =\left\|u_{1}-u_{h_{1}}^{1}\right\|_{1}
\end{aligned}
$$

or

$$
\begin{aligned}
& \text { (3) } \max \left\{\rho\left\|u_{2}-u_{h_{2}}^{1}\right\|_{2},\left\|u_{1}-u_{h_{1}}^{1}\right\|_{1}, \max _{2<j \leq m}\left\|u_{j}-u_{h_{j}}^{0}\right\|_{j}\right\} \\
& =\max _{2<j \leq m}\left\|u_{j}-u_{h_{j}}^{0}\right\|_{j} .
\end{aligned}
$$

Case 1 implies that

$$
\begin{gathered}
\left\|u_{2}-u_{h_{2}}^{1}\right\|_{2} \leq C h^{2}|\log h|+\rho\left\|u_{2}-u_{h_{2}}^{1}\right\|_{2}, \\
\left\|u_{1}-u_{h_{1}}^{1}\right\|_{1} \leq \rho\left\|u_{2}-u_{h_{2}}^{1}\right\|_{2}, \\
\max _{2<j \leq m}\left\|u_{j}-u_{h_{j}}^{0}\right\|_{j} \leq \rho\left\|u_{2}-u_{h_{2}}^{1}\right\|_{2} .
\end{gathered}
$$

Then,

$$
\begin{gathered}
\left\|u_{2}-u_{h_{2}}^{1}\right\|_{2} \leq \frac{1}{1-\rho} C h^{2}|\log h|, \\
\left\|u_{1}-u_{h_{1}}^{1}\right\|_{1} \leq \rho\left\|u_{2}-u_{h_{2}}^{1}\right\|_{1} \\
\leq \frac{\rho}{1-\rho} C h^{2}|\log h|<\frac{1}{1-\rho} C h^{2}|\log h|, \\
\max _{2<j \leq m}\left\|u_{j}-u_{h_{j}}^{0}\right\|_{j} \leq \rho\left\|u_{2}-u_{h_{2}}^{1}\right\|_{1} \\
\leq \frac{\rho}{1-\rho} C h^{2}|\log h|<C h^{2}|\log h| .
\end{gathered}
$$


We note that the two last inequalities coincide with (91) and (69), respectively. Case 2 implies that

$$
\begin{gathered}
\left\|u_{2}-u_{h_{2}}^{1}\right\|_{2} \leq C h^{2}|\log h|+\left\|u_{1}-u_{h_{1}}^{1}\right\|_{1}, \\
\rho\left\|u_{2}-u_{h_{2}}^{1}\right\|_{2} \leq\left\|u_{1}-u_{h_{1}}^{1}\right\|_{1}, \\
\max _{2<j \leq m}\left\|u_{j}-u_{h_{j}}^{0}\right\|_{j} \leq\left\|u_{1}-u_{h_{1}}^{1}\right\|_{1} .
\end{gathered}
$$

By multiplying (98) by $\rho$, we get

$$
\begin{gathered}
\rho\left\|u_{2}-u_{h_{2}}^{1}\right\|_{2} \leq \rho C h^{2}|\log h|+\rho\left\|u_{1}-u_{h_{1}}^{1}\right\|_{1}, \\
\rho\left\|u_{2}-u_{h_{2}}^{1}\right\|_{2} \leq\left\|u_{1}-u_{h_{1}}^{1}\right\|_{1}, \\
\max _{2<j \leq m}\left\|u_{j}-u_{h_{j}}^{0}\right\|_{j} \leq\left\|u_{1}-u_{h_{1}}^{1}\right\|_{1} .
\end{gathered}
$$

We remark that $\rho\left\|u_{2}-u_{h_{2}}^{1}\right\|_{2}$ is bounded by both $\rho C h^{2}$ $|\log h|+\rho\left\|u_{1}-u_{h_{1}}^{1}\right\|_{1}$ and $\left\|u_{1}-u_{h_{1}}^{1}\right\|_{1}$ then

(a) $\left\|u_{1}-u_{h_{1}}^{1}\right\|_{1}<\rho C h^{2}|\log h|+\rho\left\|u_{1}-u_{h_{1}}^{1}\right\|_{1}$

or

(b) $\rho C h^{2}|\log h|+\rho\left\|u_{1}-u_{h_{1}}^{1}\right\|_{1}<\left\|u_{1}-u_{h_{1}}^{1}\right\|_{1}$.

Which implies that

$$
\left\|u_{1}-u_{h_{1}}^{1}\right\|_{1}<\frac{\rho}{1-\rho} C h^{2}|\log h|
$$

or

$$
\frac{\rho}{1-\rho} C h^{2}|\log h|<\left\|u_{1}-u_{h_{1}}^{1}\right\|_{1} .
$$

That is

$$
\left\|u_{1}-u_{h_{1}}^{1}\right\|_{1}<\frac{\rho}{1-\rho} C h^{2}|\log h|<\frac{1}{1-\rho} C h^{2}|\log h|
$$

or

$$
\frac{\rho}{1-\rho} C h^{2}|\log h|<\left\|u_{1}-u_{h_{1}}^{1}\right\|_{1} \leq \frac{1}{1-\rho} C h^{2}|\log h| .
$$

It is clear that both cases (a) and (b) are true because they coincide with (91). So there is either contradiction and case 2 is impossible or cases 2 is possible and we must have

$$
\left\|u_{1}-u_{h_{1}}^{1}\right\|_{1}=\frac{\rho}{1-\rho} C h^{2}|\log h| .
$$

Then case 2 implies

$$
\begin{aligned}
\left\|u_{2}-u_{h_{2}}^{1}\right\|_{2} & \leq C h^{2}|\log h|+\left\|u_{1}-u_{h_{1}}^{1}\right\|_{1} \\
& =C h^{2}|\log h|+\frac{\rho}{1-\rho} C h^{2}|\log h| \\
& =\frac{1}{1-\rho} C h^{2}|\log h| .
\end{aligned}
$$

With

$$
\begin{aligned}
\max _{2<j \leq m}\left\|u_{j}-u_{h_{j}}^{0}\right\|_{j} & \leq\left\|u_{1}-u_{h_{1}}^{1}\right\|_{1} \\
& =\frac{\rho}{1-\rho} C h^{2}|\log h| \\
& <C h^{2}|\log h|
\end{aligned}
$$

which coincides with (69). Case 3 implies

$$
\begin{gathered}
\left\|u_{2}-u_{h_{2}}^{1}\right\|_{2} \leq C h^{2}|\log h|+\max _{2<j \leq m}\left\|u_{j}-u_{h_{j}}^{0}\right\|_{j}, \\
\rho\left\|u_{2}-u_{h_{2}}^{1}\right\|_{2} \leq \max _{2<j \leq m}\left\|u_{j}-u_{h_{j}}^{0}\right\|_{j}, \\
\left\|u_{1}-u_{h_{1}}^{1}\right\|_{1} \leq \max _{2<j \leq m}\left\|u_{j}-u_{h_{j}}^{0}\right\|_{j} .
\end{gathered}
$$

By multiplying (109) by $\rho$ we get

$$
\begin{gathered}
\rho\left\|u_{2}-u_{h_{2}}^{1}\right\|_{2} \leq \rho C h^{2}|\log h|+\rho \max _{2<j \leq m}\left\|u_{j}-u_{h_{j}}^{0}\right\|_{j}, \\
\rho\left\|u_{2}-u_{h_{2}}^{1}\right\|_{2} \leq \max _{2<j \leq m}\left\|u_{j}-u_{h_{j}}^{0}\right\|_{j}, \\
\left\|u_{1}-u_{h_{1}}^{1}\right\|_{1} \leq \max _{2<j \leq m}\left\|u_{j}-u_{h_{j}}^{0}\right\|_{j} .
\end{gathered}
$$

So $\rho\left\|u_{2}-u_{h_{2}}^{1}\right\|_{2}$ is bounded by both $\rho C h^{2}|\log h|+\rho \max _{2<j \leq m}$ $\left\|u_{j}-u_{h_{j}}^{0}\right\|_{j}$ and $\max _{2<j \leq m}\left\|u_{j}-u_{h_{j}}^{0}\right\|_{j}$ then $w$ have

(c) $\max _{2<j \leq m}\left\|u_{j}-u_{h_{j}}^{0}\right\|_{j}<\rho C h^{2}|\log h|+\rho \max _{2<j \leq m}\left\|u_{j}-u_{h_{j}}^{0}\right\|_{j}$

or

(d) $\rho C h^{2}|\log h|+\rho \max _{2<j \leq m}\left\|u_{j}-u_{h_{j}}^{0}\right\|_{j}<\max _{2<j \leq m}\left\|u_{j}-u_{h_{j}}^{0}\right\|_{j}$.

Which implies

$$
\max _{2<j \leq m}\left\|u_{j}-u_{h_{j}}^{0}\right\|_{j}<\frac{\rho}{1-\rho} C h^{2}|\log h|
$$

or

$$
\frac{\rho}{1-\rho} C h^{2}|\log h|<\max _{2<j \leq m}\left\|u_{j}-u_{h_{j}}^{0}\right\|_{j}
$$

That is,

$$
\max _{2<j \leq m}\left\|u_{j}-u_{h_{j}}^{0}\right\|_{j}<\frac{\rho}{1-\rho} C h^{2}|\log h|<C h^{2}|\log h|
$$

or

$$
\frac{\rho}{1-\rho} C h^{2}|\log h|<\max _{2<j \leq m}\left\|u_{j}-u_{h_{j}}^{0}\right\|_{j}<C h^{2}|\log h| .
$$


It is clear that both cases (c) and (d) are true because they coincide with (69). So there is contradiction and case 3 is impossible or case 3 is possible only if

$$
\max _{2<j \leq m}\left\|u_{j}-u_{h_{j}}^{0}\right\|_{j}=\frac{\rho}{1-\rho} C h^{2}|\log h| .
$$

Then case 3 implies that

$$
\begin{aligned}
\left\|u_{2}-u_{h_{2}}^{1}\right\|_{2} & \leq C h^{2}|\log h|+\max _{2<j \leq m}\left\|u_{j}-u_{h_{j}}^{0}\right\|_{j} \\
& =C h^{2}|\log h|+\frac{\rho}{1-\rho} C h^{2}|\log h| \\
& =\frac{1}{1-\rho} C h^{2}|\log h|,
\end{aligned}
$$

with

$$
\left\|u_{1}-u_{h_{1}}^{1}\right\|_{1} \leq \max _{2<j \leq m}\left\|u_{j}-u_{h_{j}}^{0}\right\|_{j}=\frac{\rho}{1-\rho} C h^{2}|\log h| .
$$

Hence, in the three cases 1,2 , and 3 we get

$$
\left\|u_{2}-u_{h_{2}}^{1}\right\|_{2} \leq \frac{1}{1-\rho} C h^{2}|\log h|
$$

Equations (91) and (120) imply

$$
\max _{1 \leq i<3}\left\|u_{i}-u_{h_{i}}^{1}\right\|_{i} \leq \frac{1}{1-\rho} C h^{2}|\log h| .
$$

Similarly applying the same idea for the rest of subdomain $\Omega_{i}, i=3, \ldots, m$, orderly, we get

$$
\left\|u_{i}-u_{h_{i}}^{1}\right\|_{i} \leq \frac{1}{1-\rho} C h^{2}|\log h| .
$$

Now, let us assume that

$$
\left\|u_{i}-u_{h_{i}}^{n}\right\|_{i} \leq \frac{1}{1-\rho} C h^{2}|\log h|, \quad i=1, \ldots, m
$$

and prove that

$$
\left\|u_{i}-u_{h_{i}}^{n+1}\right\|_{i} \leq \frac{1}{1-\rho} C h^{2}|\log h|, \quad i=1, \ldots, m .
$$

Equation (124) is obtained sequentially in the order of the numbering of subdomains. We begin by subdomain 1 ; indeed applying (73) to subdomain one, for iteration $n+1$, we get

$$
\begin{aligned}
\left\|u_{1}-u_{h_{1}}^{n+1}\right\|_{1} \leq & C h^{2}|\log h| \\
& +\max \left\{\rho\left\|u_{1}-u_{h_{1}}^{n+1}\right\|_{1}, \max _{1<j \leq m}\left\|u_{j}-u_{h_{j}}^{n}\right\|_{j}\right\}
\end{aligned}
$$

We have to distinguish the two following cases

(1) $\max \left\{\rho\left\|u_{1}-u_{h_{1}}^{n+1}\right\|_{1}, \max _{1<j \leq m}\left\|u_{j}-u_{h_{j}}^{n}\right\|_{j}\right\}=\rho\left\|u_{1}-u_{h_{1}}^{n+1}\right\|_{1}$ or

$$
\begin{aligned}
& \text { (2) } \max \left\{\rho\left\|u_{1}-u_{h_{1}}^{n+1}\right\|_{1}, \max _{1<j \leq m}\left\|u_{j}-u_{h_{j}}^{n}\right\|_{j}\right\} \\
& =\max _{1<j \leq m}\left\|u_{j}-u_{h_{j}}^{n}\right\|_{j} .
\end{aligned}
$$

Case 1 implies

$$
\begin{gathered}
\left\|u_{1}-u_{h_{1}}^{n+1}\right\|_{1} \leq C h^{2}|\log h|+\rho\left\|u_{1}-u_{h_{1}}^{n+1}\right\|_{1}, \\
\max _{1<j \leq m}\left\|u_{j}-u_{h_{j}}^{n}\right\|_{j} \leq \rho\left\|u_{1}-u_{h_{1}}^{n+1}\right\|_{1} .
\end{gathered}
$$

Then

$$
\begin{gathered}
\left\|u_{1}-u_{h_{1}}^{n+1}\right\|_{1} \leq \frac{1}{1-\rho} C h^{2}|\log h| \\
\max _{1<j \leq m}\left\|u_{j}-u_{h_{j}}^{n}\right\|_{j} \leq \rho\left\|u_{1}-u_{h_{1}}^{n+1}\right\|_{1} \\
\leq \frac{\rho}{1-\rho} C h^{2}|\log h|<\frac{1}{1-\rho} C h^{2}|\log h| .
\end{gathered}
$$

Case 2 implies

$$
\begin{gathered}
\left\|u_{1}-u_{h_{1}}^{n+1}\right\|_{1} \leq C h^{2}|\log h|+\max _{1<j \leq m}\left\|u_{j}-u_{h_{j}}^{n}\right\|_{j}, \\
\rho\left\|u_{1}-u_{h_{1}}^{n+1}\right\|_{1} \leq \max _{1<j \leq m}\left\|u_{j}-u_{h_{j}}^{n}\right\|_{j} .
\end{gathered}
$$

By multiplying (130) by $\rho$ we get

$$
\begin{gathered}
\rho\left\|u_{1}-u_{h_{1}}^{n+1}\right\|_{1} \leq \rho C h^{2}|\log h|+\rho \max _{1<j \leq m}\left\|u_{j}-u_{h_{j}}^{n}\right\|_{j}, \\
\rho\left\|u_{1}-u_{h_{1}}^{n+1}\right\|_{1} \leq \max _{1<j \leq m}\left\|u_{j}-u_{h_{j}}^{n}\right\|_{j}
\end{gathered}
$$

So $\rho\left\|u_{1}-u_{h_{1}}^{n+1}\right\|_{1}$ is bounded by both $\rho C h^{2}|\log h|+\rho \max _{1<j \leq m}$ $\left\|u_{j}-u_{h_{j}}^{n}\right\|_{j}$ and $\max _{1<j \leq m}\left\|u_{j}-u_{h_{j}}^{n}\right\|_{j}$ then

$$
\text { (a) } \max _{1<j \leq m}\left\|u_{j}-u_{h_{j}}^{n}\right\|_{j}<\rho C h^{2}|\log h|+\rho \max _{1<j \leq m}\left\|u_{j}-u_{h_{j}}^{n}\right\|_{j}
$$

or

(b) $\rho C h^{2}|\log h|+\rho \max _{1<j \leq m}\left\|u_{j}-u_{h_{j}}^{n}\right\|_{j}<\max _{1<j \leq m}\left\|u_{j}-u_{h_{j}}^{n}\right\|_{j}$.

That is

$$
\max _{1<j \leq m}\left\|u_{j}-u_{h_{j}}^{n}\right\|_{j}<\frac{\rho}{1-\rho} C h^{2}|\log h|
$$

or

$$
\frac{\rho}{1-\rho} C h^{2}|\log h|<\max _{1<j \leq m}\left\|u_{j}-u_{h_{j}}^{n}\right\|_{j} .
$$


Which implies

$$
\max _{1<j \leq m}\left\|u_{j}-u_{h_{j}}^{n}\right\|_{j}<\frac{\rho}{1-\rho} C h^{2}|\log h|<\frac{1}{1-\rho} C h^{2}|\log h|
$$

or

$$
\frac{\rho}{1-\rho} C h^{2}|\log h|<\max _{1<j \leq m}\left\|u_{j}-u_{h_{j}}^{n}\right\|_{j}<\frac{1}{1-\rho} C h^{2}|\log h| .
$$

It is clear that both cases (a) and (b) are true because both coincide with (123). So either there is a contradiction and thus case 2 is impossible or case 2 is possible only if

$$
\max _{1<j \leq m}\left\|u_{j}-u_{h_{j}}^{n}\right\|_{j}=\frac{\rho}{1-\rho} C h^{2}|\log h| .
$$

Then case 2 implies

$$
\begin{aligned}
\left\|u_{1}-u_{h_{1}}^{n+1}\right\|_{1} & \leq C h^{2}|\log h|+\max _{1<j \leq m}\left\|u_{j}-u_{h_{j}}^{n}\right\|_{j} \\
& =C h^{2}|\log h|+\frac{\rho}{1-\rho} C h^{2}|\log h| \\
& =\frac{1}{1-\rho} C h^{2}|\log h| .
\end{aligned}
$$

Hence in both cases 1 and 2 we obtain

$$
\left\|u_{1}-u_{h_{1}}^{n+1}\right\|_{1} \leq \frac{1}{1-\rho} C h^{2}|\log h| .
$$

Similarly applying (73) to the second subdomain, for iteration $n+1$, we get

$$
\begin{aligned}
&\left\|u_{1}-u_{h_{2}}^{n+1}\right\|_{2} \leq C h^{2}|\log h| \\
&+\max \left\{\rho\left\|u_{2}-u_{h_{2}}^{n+1}\right\|_{2},\left\|u_{1}-u_{h_{1}}^{n+}\right\|_{1},\right. \\
&\left.\max _{2<j \leq m}\left\|u_{j}-u_{h_{j}}^{n}\right\|_{j}\right\} .
\end{aligned}
$$

We have to distinguish the three following cases

$$
\begin{aligned}
& \text { (1) } \max \left\{\rho\left\|u_{2}-u_{h_{2}}^{n+1}\right\|_{2},\left\|u_{1}-u_{h_{1}}^{n+1}\right\|_{1}, \max _{2<j \leq m}\left\|u_{j}-u_{h_{j}}^{n}\right\|_{j}\right\} \\
& =\rho\left\|u_{2}-u_{h_{2}}^{n+1}\right\|_{2}
\end{aligned}
$$

or

$$
\begin{aligned}
& \text { (2) } \max \left\{\rho\left\|u_{2}-u_{h_{2}}^{n+1}\right\|_{2},\left\|u_{1}-u_{h_{1}}^{n+1}\right\|_{1}, \max _{2<j \leq m}\left\|u_{j}-u_{h_{j}}^{n}\right\|_{j}\right\} \\
& =\left\|u_{1}-u_{h_{1}}^{n+1}\right\|_{1}
\end{aligned}
$$

or

$$
\begin{aligned}
& \text { (3) } \max \left\{\rho\left\|u_{2}-u_{h_{2}}^{n+1}\right\|_{2},\left\|u_{1}-u_{h_{1}}^{n+1}\right\|_{1}, \max _{2<j \leq m}\left\|u_{j}-u_{h_{j}}^{n}\right\|_{j}\right\} \\
& =\max _{2<j \leq m}\left\|u_{j}-u_{h_{j}}^{n}\right\|_{j} .
\end{aligned}
$$

Case 1 implies

$$
\begin{gathered}
\left\|u_{2}-u_{h_{2}}^{n+1}\right\|_{2} \leq C h^{2}|\log h|+\rho\left\|u_{2}-u_{h_{2}}^{n+1}\right\|_{2}, \\
\left\|u_{1}-u_{h_{1}}^{n+1}\right\|_{1} \leq \rho\left\|u_{2}-u_{h_{2}}^{n+1}\right\|_{2}, \\
\max _{2<j \leq m}\left\|u_{j}-u_{h_{j}}^{n}\right\|_{j} \leq \rho\left\|u_{2}-u_{h_{2}}^{n+1}\right\|_{2} .
\end{gathered}
$$

Then

$$
\begin{gathered}
\left\|u_{2}-u_{h_{2}}^{n+1}\right\|_{2} \leq \frac{1}{1-\rho} C h^{2}|\log h| \\
\left\|u_{1}-u_{h_{1}}^{n+1}\right\|_{1} \leq \rho\left\|u_{2}-u_{h_{2}}^{n+1}\right\|_{2} \\
\leq \frac{\rho}{1-\rho} C h^{2}|\log h|<\frac{1}{1-\rho} C h^{2}|\log h|, \\
\max _{2<j \leq m}\left\|u_{j}-u_{h_{j}}^{n}\right\|_{j} \leq \rho\left\|u_{2}-u_{h_{2}}^{n+1}\right\|_{2} \\
\leq \frac{\rho}{1-\rho} C h^{2}|\log h|<\frac{1}{1-\rho} C h^{2}|\log h| .
\end{gathered}
$$

We note that the two last inequalities coincide with (140) and (123) respectively. Case 2 implies that

$$
\begin{gathered}
\left\|u_{2}-u_{h_{2}}^{n+1}\right\|_{2} \leq C h^{2}|\log h|+\left\|u_{1}-u_{h_{1}}^{n+1}\right\|_{1}, \\
\rho\left\|u_{2}-u_{h_{2}}^{n+1}\right\|_{2} \leq\left\|u_{1}-u_{h_{1}}^{n+1}\right\|_{1}, \\
\max _{2<j \leq m}\left\|u_{j}-u_{h_{j}}^{n}\right\|_{j} \leq\left\|u_{1}-u_{h_{1}}^{n+1}\right\|_{1} .
\end{gathered}
$$

By multiplying (147) by $\rho$ we get

$$
\begin{gathered}
\rho\left\|u_{2}-u_{h_{2}}^{n+1}\right\|_{2} \leq \rho C h^{2}|\log h|+\rho\left\|u_{1}-u_{h_{1}}^{n+1}\right\|_{1}, \\
\rho\left\|u_{2}-u_{h_{2}}^{n+1}\right\|_{2} \leq\left\|u_{1}-u_{h_{1}}^{n+1}\right\|_{1}, \\
\max _{2<j \leq m}\left\|u_{j}-u_{h_{j}}^{n}\right\|_{j} \leq\left\|u_{1}-u_{h_{1}}^{n+1}\right\|_{1} .
\end{gathered}
$$

We remark that $\rho\left\|u_{2}-u_{h_{2}}^{n+1}\right\|_{2}$ is bounded by both $\rho C h^{2}$ $|\log h|+\rho\left\|u_{1}-u_{h_{1}}^{n+1}\right\|_{1}$ and $\left\|u_{1}-u_{h_{1}}^{n+1}\right\|_{1}$ then

(a) $\left\|u_{1}-u_{h_{1}}^{n+1}\right\|_{1}<\rho C h^{2}|\log h|+\rho\left\|u_{1}-u_{h_{1}}^{n+1}\right\|_{1}$

or

(b) $\rho C h^{2}|\log h|+\rho\left\|u_{1}-u_{h_{1}}^{n+1}\right\|_{1}<\left\|u_{1}-u_{h_{1}}^{n+1}\right\|_{1}$. 
Which implies

$$
\left\|u_{1}-u_{h_{1}}^{n+1}\right\|_{1}<\frac{\rho}{1-\rho} C h^{2}|\log h|
$$

or

$$
\frac{\rho}{1-\rho} C h^{2}|\log h|<\left\|u_{1}-u_{h_{1}}^{n+1}\right\|_{1} .
$$

That is

$$
\left\|u_{1}-u_{h_{1}}^{n+1}\right\|_{1}<\frac{\rho}{1-\rho} C h^{2}|\log h|<\frac{1}{1-\rho} C h^{2}|\log h|
$$

or

$$
\frac{\rho}{1-\rho} C h^{2}|\log h|<\left\|u_{1}-u_{h_{1}}^{n+1}\right\|_{1} \leq \frac{1}{1-\rho} C h^{2}|\log h| .
$$

It is clear that both cases are true because they both coincide with (140). So there is either contradiction and case 2 is impossible or cases 2 is possible and we must have

$$
\left\|u_{1}-u_{h_{1}}^{n+1}\right\|_{1}=\frac{\rho}{1-\rho} C h^{2}|\log h| .
$$

Case 2 implies that

$$
\begin{aligned}
\left\|u_{2}-u_{h_{2}}^{n+1}\right\|_{2} & \leq C h^{2}|\log h|+\left\|u_{1}-u_{h_{1}}^{n+1}\right\|_{1} \\
& =C h^{2}|\log h|+\frac{\rho}{1-\rho} C h^{2}|\log h| \\
& =\frac{1}{1-\rho} C h^{2}|\log h| .
\end{aligned}
$$

With

$$
\begin{aligned}
\max _{2<j \leq m}\left\|u_{j}-u_{h_{j}}^{n}\right\|_{j} & \leq\left\|u_{1}-u_{h_{1}}^{n+1}\right\|_{1} \\
& =\frac{\rho}{1-\rho} C h^{2}|\log h| \\
& <\frac{1}{1-\rho} C h^{2}|\log h|
\end{aligned}
$$

which coincides with (123). Case 3 implies

$$
\begin{gathered}
\left\|u_{2}-u_{h_{2}}^{n+1}\right\|_{2} \leq C h^{2}|\log h|+\max _{2<j \leq m}\left\|u_{j}-u_{h_{j}}^{n}\right\|_{j}, \\
\rho\left\|u_{2}-u_{h_{2}}^{n+1}\right\|_{2} \leq \max _{2<j \leq m}\left\|u_{j}-u_{h_{j}}^{n}\right\|_{j}, \\
\left\|u_{1}-u_{h_{1}}^{n+1}\right\|_{1} \leq \max _{2<j \leq m}\left\|u_{j}-u_{h_{j}}^{n}\right\|_{j} .
\end{gathered}
$$

By multiplying (158) by $\rho$ we get

$$
\begin{gathered}
\rho\left\|u_{2}-u_{h_{2}}^{n+1}\right\|_{2} \leq \rho C h^{2}|\log h|+\rho \max _{2<j \leq m}\left\|u_{j}-u_{h_{j}}^{n}\right\|_{j}, \\
\rho\left\|u_{2}-u_{h_{2}}^{n+1}\right\|_{2} \leq \max _{2<j \leq m}\left\|u_{j}-u_{h_{j}}^{n}\right\|_{j}, \\
\left\|u_{1}-u_{h_{1}}^{n+1}\right\|_{1} \leq \max _{2<j \leq m}\left\|u_{j}-u_{h_{j}}^{n}\right\|_{j} .
\end{gathered}
$$

So $\rho\left\|u_{2}-u_{h_{2}}^{n+1}\right\|_{2}$ is bounded by both $\rho C h^{2}|\log h|+\rho \max _{2<j \leq m}$ $\left\|u_{j}-u_{h_{j}}^{n}\right\|_{j}$ and $\max _{2<j \leq m}\left\|u_{j}-u_{h_{j}}^{n}\right\|_{j}$ then

(c) $\max _{2<j \leq m}\left\|u_{j}-u_{h_{j}}^{n}\right\|_{j}<\rho C h^{2}|\log h|+\rho \max _{2<j \leq m}\left\|u_{j}-u_{h_{j}}^{n}\right\|_{j}$

or

(d) $\rho C h^{2}|\log h|+\rho \max _{2<j \leq m}\left\|u_{j}-u_{h_{j}}^{n}\right\|_{j}<\max _{2<j \leq m}\left\|u_{j}-u_{h_{j}}^{n}\right\|_{j}$.

Which implies

$$
\max _{2<j \leq m}\left\|u_{j}-u_{h_{j}}^{n}\right\|_{j}<\frac{\rho}{1-\rho} C h^{2}|\log h|
$$

or

$$
\frac{\rho}{1-\rho} C h^{2}|\log h|<\max _{2<j \leq m}\left\|u_{j}-u_{h_{j}}^{n}\right\|_{j} .
$$

That is

$$
\max _{2<j \leq m}\left\|u_{j}-u_{h_{j}}^{n}\right\|_{j}<\frac{\rho}{1-\rho} C h^{2}|\log h|<\frac{1}{1-\rho} C h^{2}|\log h|
$$

or

$$
\frac{\rho}{1-\rho} C h^{2}|\log h|<\max _{2<j \leq m}\left\|u_{j}-u_{h_{j}}^{n}\right\|_{j} \leq \frac{1}{1-\rho} C h^{2}|\log h| .
$$

It is clear that both cases (c) and (d) are true because they both coincide with (123). So there is contradiction and case 3 is impossible or case 3 is possible only if

$$
\max _{2<j \leq m}\left\|u_{j}-u_{h_{j}}^{n}\right\|_{j}=\frac{\rho}{1-\rho} C h^{2}|\log h| .
$$

Then case 3 implies

$$
\begin{aligned}
\left\|u_{2}-u_{h_{2}}^{n+1}\right\|_{2} & \leq C h^{2}|\log h|+\max _{2<j \leq m}\left\|u_{j}-u_{h_{j}}^{n}\right\|_{j} \\
& =C h^{2}|\log h|+\frac{\rho}{1-\rho} C h^{2}|\log h| \\
& =\frac{1}{1-\rho} C h^{2}|\log h| .
\end{aligned}
$$

With

$$
\left\|u_{1}-u_{h_{1}}^{n+1}\right\|_{1} \leq \max _{2<j \leq m}\left\|u_{j}-u_{h_{j}}^{n}\right\|_{j}=\frac{\rho}{1-\rho} C h^{2}|\log h| .
$$

Hence in three cases 1,2, and 3 we get

$$
\left\|u_{2}-u_{h_{2}}^{n+1}\right\|_{2} \leq \frac{1}{1-\rho} C h^{2}|\log h| .
$$

Equations (140) and (169) imply

$$
\max _{1 \leq j<3}\left\|u_{j}-u_{h_{j}}^{n+1}\right\|_{j} \leq \frac{1}{1-\rho} C h^{2}|\log h| .
$$


Similarly applying the same idea for the rest of subdomains $\Omega_{i}, i=3, \ldots, m$, orderly we get

$$
\left\|u_{i}-u_{h_{i}}^{n+1}\right\|_{i} \leq \frac{1}{1-\rho} C h^{2}|\log h|
$$

which is the desired result. In the sequel we deal with the second subinterval $[1 / 2,1[$, indeed $\rho \in[1 / 2,1[$ so

$$
\frac{\rho}{1-\rho} \geq 1
$$

For $n=0$, applying (73) to subdomain one we get

$$
\begin{aligned}
\left\|u_{1}-u_{h_{1}}^{1}\right\|_{1} \leq & C h^{2}|\log h| \\
& +\max \left\{\rho\left\|u_{1}-u_{h_{1}}^{1}\right\|_{1}, \max _{1<j \leq m}\left\|u_{j}-u_{h_{j}}^{0}\right\|_{j}\right\} .
\end{aligned}
$$

We have to distinguish the two following cases

$$
\text { (1) } \max \left\{\rho\left\|u_{1}-u_{h_{1}}^{1}\right\|_{1}, \max _{1<j \leq m}\left\|u_{j}-u_{h_{j}}^{0}\right\|_{j}\right\}=\rho\left\|u_{1}-u_{h_{1}}^{1}\right\|_{1}
$$

or

$$
\begin{aligned}
& \text { (2) } \max \left\{\rho\left\|u_{1}-u_{h_{1}}^{1}\right\|_{1}, \max _{1<j \leq m}\left\|u_{j}-u_{h_{j}}^{0}\right\|_{j}\right\} \\
& =\max _{1<j \leq m}\left\|u_{j}-u_{h_{j}}^{0}\right\|_{j} .
\end{aligned}
$$

Case 1 implies

$$
\begin{gathered}
\left\|u_{1}-u_{h_{1}}^{1}\right\|_{1} \leq C h^{2}|\log h|+\rho\left\|u_{1}-u_{h_{1}}^{1}\right\|_{1}, \\
\max _{1<j \leq m}\left\|u_{j}-u_{h_{j}}^{0}\right\|_{j} \leq \rho\left\|u_{1}-u_{h_{1}}^{1}\right\|_{1} .
\end{gathered}
$$

Then

$$
\begin{gathered}
\left\|u_{1}-u_{h_{1}}^{1}\right\|_{1} \leq \frac{1}{1-\rho} C h^{2}|\log h|, \\
\max _{1<j \leq m}\left\|u_{j}-u_{h_{j}}^{0}\right\|_{j} \leq \rho\left\|u_{1}-u_{h_{1}}^{1}\right\|_{1} \leq \frac{\rho}{1-\rho} C h^{2}|\log h| .
\end{gathered}
$$

Case 2 implies

$$
\begin{gathered}
\left\|u_{1}-u_{h_{1}}^{1}\right\|_{1} \leq C h^{2}|\log h|+\max _{1<j \leq m}\left\|u_{j}-u_{h_{j}}^{0}\right\|_{j}, \\
\rho\left\|u_{1}-u_{h_{1}}^{1}\right\|_{1} \leq \max _{1<j \leq m}\left\|u_{j}-u_{h_{j}}^{0}\right\|_{j} .
\end{gathered}
$$

By multiplying (178) by $\rho$ we get

$$
\begin{gathered}
\rho\left\|u_{1}-u_{h_{1}}^{1}\right\|_{1} \leq \rho C h^{2}|\log h|+\rho \max _{1<j \leq m}\left\|u_{j}-u_{h_{j}}^{0}\right\|_{j}, \\
\rho\left\|u_{1}-u_{h_{1}}^{1}\right\|_{1} \leq \max _{1<j \leq m}\left\|u_{j}-u_{h_{j}}^{0}\right\|_{j}
\end{gathered}
$$

So $\rho\left\|u_{1}-u_{h_{1}}^{1}\right\|_{1}$ is bounded by both $\rho C h^{2}|\log h|+\rho \max _{1<j \leq m}$ $\left\|u_{j}-u_{h_{j}}^{0}\right\|_{j}$ and $\max _{1<j \leq m}\left\|u_{j}-u_{h_{j}}^{0}\right\|_{j}$ then

(a) $\max _{1<j \leq m}\left\|u_{j}-u_{h_{j}}^{0}\right\|_{j}<\rho C h^{2}|\log h|+\rho \max _{1<j \leq m}\left\|u_{j}-u_{h_{j}}^{0}\right\|_{j}$

or

(b) $\rho C h^{2}|\log h|+\rho \max _{1<j \leq m}\left\|u_{j}-u_{h_{j}}^{0}\right\|_{j}<\max _{1<j \leq m}\left\|u_{j}-u_{h_{j}}^{0}\right\|_{j}$.

That is

$$
\max _{1<j \leq m}\left\|u_{j}-u_{h_{j}}^{0}\right\|_{j}<\frac{\rho}{1-\rho} C h^{2}|\log h|
$$

or

$$
\frac{\rho}{1-\rho} C h^{2}|\log h|<\max _{1<j \leq m}\left\|u_{j}-u_{h_{j}}^{0}\right\|_{j}
$$

Which implies

$$
\max _{1<j \leq m}\left\|u_{j}-u_{h_{j}}^{0}\right\|_{j}<C h^{2}|\log h| \leq \frac{\rho}{1-\rho} C h^{2}|\log h|
$$

or

$$
\frac{\rho}{1-\rho} C h^{2}|\log h|<\max _{1<j \leq m}\left\|u_{j}-u_{h_{j}}^{0}\right\|_{j}<C h^{2}|\log h| .
$$

It is clear that only the case (a) is true because it coincides with (69) whereas (b) contradicts (172). Then case (a) with case 2 imply

$$
\begin{aligned}
\left\|u_{1}-u_{h_{1}}^{1}\right\|_{1} & \leq C h^{2}|\log h|+\max _{1<j \leq m}\left\|u_{j}-u_{h_{j}}^{0}\right\|_{j} \\
& <C h^{2}|\log h|+\frac{\rho}{1-\rho} C h^{2}|\log h| \\
& =\frac{1}{1-\rho} C h^{2}|\log h| .
\end{aligned}
$$

Hence in both cases 1 and 2 we get

$$
\left\|u_{1}-u_{h_{1}}^{1}\right\|_{1} \leq \frac{1}{1-\rho} C h^{2}|\log h| .
$$

By the same way we can obtain orderly

$$
\left\|u_{i}-u_{h_{i}}^{1}\right\|_{i} \leq \frac{1}{1-\rho} C h^{2}|\log h|, \quad i=2, \ldots, m
$$

The remainder of the proof is also by induction by addopting the same idea applied in iteration one.

Part 2. We deal with situation (B). We begin by the first subinterval that is $\rho \in] 0,1 / 2$ [; Indeed for $n=0$, (73) implies for subdomain 1 ,

$$
\begin{aligned}
\left\|u_{1}-u_{h_{1}}^{1}\right\|_{1} \leq & C h^{2}|\log h| \\
& +\max \left\{\rho\left\|u_{1}-u_{h_{1}}^{1}\right\|_{1}, \max _{1<j}\left\|u_{j}-u_{h_{j}}^{0}\right\|_{j}\right\} .
\end{aligned}
$$


We have to distinguish the two following cases

$$
\text { (1) } \max \left\{\rho\left\|u_{1}-u_{h_{1}}^{1}\right\|_{1}, \max _{1<j}\left\|u_{j}-u_{h_{j}}^{0}\right\|_{j}\right\}=\rho\left\|u_{1}-u_{h_{1}}^{1}\right\|_{1}
$$

or

(2) $\max \left\{\rho\left\|u_{1}-u_{h_{1}}^{1}\right\|_{1}, \max _{1<j}\left\|u_{j}-u_{h_{j}}^{0}\right\|_{j}\right\}=\max _{1<j}\left\|u_{j}-u_{h_{j}}^{0}\right\|_{j}$.

Case 1 implies

$$
\begin{gathered}
\left\|u_{1}-u_{h_{1}}^{1}\right\|_{1} \leq C h^{2}|\log h|+\rho\left\|u_{1}-u_{h_{1}}^{1}\right\|_{1}, \\
\max _{1<j}\left\|u_{j}-u_{h_{j}}^{0}\right\|_{j} \leq \rho\left\|u_{1}-u_{h_{1}}^{1}\right\|_{1} .
\end{gathered}
$$

So

$$
\begin{aligned}
&\left\|u_{1}-u_{h_{1}}^{1}\right\|_{1} \leq \frac{1}{1-\rho} C h^{2}|\log h|, \\
& \max _{1<j}\left\|u_{j}-u_{h_{j}}^{0}\right\|_{j} \leq \rho\left\|u_{1}-u_{h_{1}}^{1}\right\|_{1} \\
& \leq \frac{\rho}{1-\rho} C h^{2}|\log h| \\
&<C h^{2}|\log h| .
\end{aligned}
$$

We remark that (193) contradicts (70) so case 1 is impossible. Case 2 implies

$$
\begin{gathered}
\left\|u_{1}-u_{h_{1}}^{1}\right\|_{1} \leq C h^{2}|\log h|+\max _{1<j}\left\|u_{j}-u_{h_{j}}^{0}\right\|_{j}, \\
\rho\left\|u_{1}-u_{h_{1}}^{1}\right\|_{1} \leq \max _{1<j}\left\|u_{j}-u_{h_{j}}^{0}\right\|_{j}
\end{gathered}
$$

By multiplying (194) by $\rho$ we get

$$
\begin{gathered}
\rho\left\|u_{1}-u_{h_{1}}^{1}\right\|_{1} \leq \rho C h^{2}|\log h|+\rho \max _{1<j}\left\|u_{j}-u_{h_{j}}^{0}\right\|_{j}, \\
\rho\left\|u_{1}-u_{h_{1}}^{1}\right\|_{1} \leq \max _{1<j}\left\|u_{j}-u_{h_{j}}^{0}\right\|_{j} \cdot
\end{gathered}
$$

It is clear that $\rho\left\|u_{1}-u_{h_{1}}^{1}\right\|_{1}$ is bounded by both $\rho C h^{2}|\log h|+$ $\rho \max _{1<j}\left\|u_{j}-u_{h_{j}}^{0}\right\|_{j}$ and $\max _{1<j}\left\|u_{j}-u_{h_{j}}^{0}\right\|_{j}$, so

$$
\text { (a) } \max _{1<j}\left\|u_{j}-u_{h_{j}}^{0}\right\|_{j}<\rho C h^{2}|\log h|+\rho \max _{1<j}\left\|u_{j}-u_{h_{j}}^{0}\right\|_{j}
$$

or

(b) $\rho C h^{2}|\log h|+\rho \max _{1<j}\left\|u_{j}-u_{h_{j}}^{0}\right\|_{j}<\max _{1<j}\left\|u_{j}-u_{h_{j}}^{0}\right\|_{j}$.

Which implies

$$
\max _{1<j}\left\|u_{j}-u_{h_{j}}^{0}\right\|_{j}<\frac{\rho}{1-\rho} C h^{2}|\log h|
$$

or

$$
\frac{\rho}{1-\rho} C h^{2}|\log h|<\max _{1<j}\left\|u_{j}-u_{h_{j}}^{0}\right\|_{j}
$$

Then

$$
C h^{2}|\log h| \leq \max _{1<j}\left\|u_{j}-u_{h_{j}}^{0}\right\|_{j}<\frac{\rho}{1-\rho} C h^{2}|\log h|
$$

or

$$
\frac{\rho}{1-\rho} C h^{2}|\log h|<C h^{2}|\log h| \leq \max _{1<j}\left\|u_{j}-u_{h_{j}}^{0}\right\|_{j} .
$$

We can see that only case (b) is possible and by adding $C h^{2}|\log h|$ in (199) we get

$$
\begin{aligned}
& C h^{2}|\log h|+\frac{\rho}{1-\rho} C h^{2}|\log h| \\
& \quad<C h^{2}|\log h|+\max _{1<j}\left\|u_{j}-u_{h_{j}}^{0}\right\|_{j}
\end{aligned}
$$

thus

$$
\frac{1}{1-\rho} C h^{2}|\log h|<C h^{2}|\log h|+\max _{1<j}\left\|u_{j}-u_{h_{j}}^{0}\right\|_{j}
$$

comparing this last inequalty (203) with (194), we deduce the two following possibilities

$$
\left\|u_{1}-u_{h_{1}}^{1}\right\|_{1}<\frac{1}{1-\rho} C h^{2}|\log h|
$$

or

$$
\frac{1}{1-\rho} C h^{2}|\log h|<\left\|u_{1}-u_{h_{1}}^{1}\right\|_{1}
$$

which implies

$$
\begin{aligned}
\left\|u_{1}-u_{h_{1}}^{1}\right\|_{1} & <\frac{1}{1-\rho} C h^{2}|\log h| \\
& <C h^{2}|\log h|+\max _{1<j}\left\|u_{j}-u_{h_{j}}^{0}\right\|_{j}
\end{aligned}
$$

or

$$
\begin{aligned}
\frac{1}{1-\rho} C h^{2}|\log h| & <\left\|u_{1}-u_{h_{1}}^{1}\right\|_{1} \\
& \leq C h^{2}|\log h|+\max _{1<j}\left\|u_{j}-u_{h_{j}}^{0}\right\|_{j}
\end{aligned}
$$

It is clear that the two possibilities are true so either there is a contradiction and case 2 is impossible or case 2 is possible only if

$$
\left\|u_{1}-u_{h_{1}}^{1}\right\|_{1}=\frac{1}{1-\rho} C h^{2}|\log h|
$$

Similarly applying (73) to subdomain 2 we get

$$
\begin{aligned}
&\left\|u_{2}-u_{h_{2}}^{1}\right\|_{2} \leq C h^{2}|\log h| \\
&+\max \left\{\rho\left\|u_{2}-u_{h_{2}}^{1}\right\|_{2},\left\|u_{1}-u_{h_{1}}^{1}\right\|_{1},\right. \\
&\left.\max _{2<j}\left\|u_{j}-u_{h_{j}}^{0}\right\|_{j}\right\} .
\end{aligned}
$$


So we have to distinguish between the three following cases

$$
\begin{aligned}
& \text { (1) } \max \left\{\rho\left\|u_{2}-u_{h_{2}}^{1}\right\|_{2},\left\|u_{1}-u_{h_{1}}^{1}\right\|_{1}, \max _{2<j}\left\|u_{j}-u_{h_{j}}^{0}\right\|_{j}\right\} \\
& =\rho\left\|u_{2}-u_{h_{2}}^{1}\right\|_{2}
\end{aligned}
$$

or

(2) $\max \left\{\rho\left\|u_{2}-u_{h_{2}}^{1}\right\|_{2},\left\|u_{1}-u_{h_{1}}^{1}\right\|_{1}, \max _{2<j}\left\|u_{j}-u_{h_{j}}^{0}\right\|_{j}\right\}$

$$
=\left\|u_{1}-u_{h_{1}}^{1}\right\|_{1}
$$

or

$$
\begin{aligned}
& \text { (3) } \max \left\{\rho\left\|u_{2}-u_{h_{2}}^{1}\right\|_{2},\left\|u_{1}-u_{h_{1}}^{1}\right\|_{1}, \max _{2<j}\left\|u_{j}-u_{h_{j}}^{0}\right\|_{j}\right\} \\
& =\max _{2<j}\left\|u_{j}-u_{h_{j}}^{0}\right\|_{j} .
\end{aligned}
$$

Case 1 implies

$$
\begin{gathered}
\left\|u_{2}-u_{h_{2}}^{1}\right\|_{2} \leq C h^{2}|\log h|+\rho\left\|u_{2}-u_{h_{2}}^{1}\right\|_{2}, \\
\left\|u_{1}-u_{h_{1}}^{1}\right\|_{1} \leq \rho\left\|u_{2}-u_{h_{2}}^{1}\right\|_{2}, \\
\max _{2<j}\left\|u_{j}-u_{h_{j}}^{0}\right\|_{j} \leq \rho\left\|u_{2}-u_{h_{2}}^{1}\right\|_{2} .
\end{gathered}
$$

Then

$$
\begin{gathered}
\left\|u_{2}-u_{h_{2}}^{1}\right\|_{2} \leq \frac{1}{1-\rho} C h^{2}|\log h| \\
\frac{1}{1-\rho} C h^{2}|\log h|=\left\|u_{1}-u_{h_{1}}^{1}\right\|_{1} \leq \rho\left\|u_{2}-u_{h_{2}}^{1}\right\|_{2} \\
\leq \frac{\rho}{1-\rho} C h^{2}|\log h| \\
\max _{2<j}\left\|u_{j}-u_{h_{j}}^{0}\right\|_{j} \leq \rho\left\|u_{2}-u_{h_{2}}^{1}\right\|_{2} \\
\leq \frac{\rho}{1-\rho} C h^{2}|\log h|<C h^{2}|\log h| .
\end{gathered}
$$

Equations (214) contradicts (70) so case 1 is impossible. Case 2 implies

$$
\begin{gathered}
\left\|u_{2}-u_{h_{2}}^{1}\right\|_{2} \leq C h^{2}|\log h|+\left\|u_{1}-u_{h_{1}}^{1}\right\|_{1}, \\
\rho\left\|u_{2}-u_{h_{2}}^{1}\right\|_{2} \leq\left\|u_{1}-u_{h_{1}}^{1}\right\|_{1}, \\
\max _{2<j}\left\|u_{j}-u_{h_{j}}^{0}\right\|_{j} \leq\left\|u_{1}-u_{h_{1}}^{1}\right\|_{1} .
\end{gathered}
$$

By multiplying (215) by $\rho$ we get

$$
\begin{gathered}
\rho\left\|u_{2}-u_{h_{2}}^{1}\right\|_{2} \leq \rho C h^{2}|\log h|+\rho\left\|u_{1}-u_{h_{1}}^{1}\right\|_{1}, \\
\rho\left\|u_{2}-u_{h_{2}}^{1}\right\|_{2} \leq\left\|u_{1}-u_{h_{1}}^{1}\right\|_{1}, \\
\max _{2<j}\left\|u_{j}-u_{h_{j}}^{0}\right\|_{j} \leq\left\|u_{1}-u_{h_{1}}^{1}\right\|_{1} .
\end{gathered}
$$

It is clear that $\rho\left\|u_{2}-u_{h_{2}}^{1}\right\|_{2}$ is bounded by both $\rho C h^{2}|\log h|+$ $\rho\left\|u_{1}-u_{h_{1}}^{1}\right\|_{1}$ and $\left\|u_{1}-u_{h_{1}}^{1}\right\|_{1}$ so

$$
\text { (a) }\left\|u_{1}-u_{h_{1}}^{1}\right\|_{1}<\rho C h^{2}|\log h|+\rho\left\|u_{1}-u_{h_{1}}^{1}\right\|_{1}
$$

or

$$
\text { (b) } \rho C h^{2}|\log h|+\rho\left\|u_{1}-u_{h_{1}}^{1}\right\|_{1}<\left\|u_{1}-u_{h_{1}}^{1}\right\|_{1} \text {. }
$$

That is

$$
\left\|u_{1}-u_{h_{1}}^{1}\right\|_{1}<\frac{\rho}{1-\rho} C h^{2}|\log h|
$$

or

$$
\frac{\rho}{1-\rho} C h^{2}|\log h|<\left\|u_{1}-u_{h_{1}}^{1}\right\|_{1} .
$$

It is clear that only case (b) is true because it coincides with (208) and by adding $C h^{2}|\log h|$ in $(220)$ we get

$$
\begin{aligned}
\frac{1}{1-\rho} C h^{2}|\log h| & =C h^{2}|\log h|+\frac{\rho}{1-\rho} C h^{2}|\log h| \\
& <C h^{2}|\log h|+\left\|u_{1}-u_{h_{1}}^{1}\right\|_{1}
\end{aligned}
$$

comparing this last inequalty with (215), we distingush the two following possibilities

$$
\left\|u_{2}-u_{h_{2}}^{1}\right\|_{2}<\frac{1}{1-\rho} C h^{2}|\log h|
$$

or

$$
\frac{1}{1-\rho} C h^{2}|\log h|<\left\|u_{2}-u_{h_{2}}^{1}\right\|_{2}
$$

which imply

$$
\left\|u_{2}-u_{h_{2}}^{1}\right\|_{2}<\frac{1}{1-\rho} C h^{2}|\log h|<C h^{2}|\log h|+\left\|u_{1}-u_{h_{1}}^{1}\right\|_{1}
$$

or

$$
\frac{1}{1-\rho} C h^{2}|\log h|<\left\|u_{2}-u_{h_{2}}^{1}\right\|_{2} \leq C h^{2}|\log h|+\left\|u_{1}-u_{h_{1}}^{1}\right\|_{1} .
$$

It is clear that the two possibilities are true so either there is a contradiction and case 2 is impossible or case 2 is possible only if

$$
\left\|u_{2}-u_{h_{2}}^{1}\right\|_{2}=\frac{1}{1-\rho} C h^{2}|\log h|
$$

Case 3 implies

$$
\begin{gathered}
\left\|u_{2}-u_{h_{2}}^{1}\right\|_{2} \leq C h^{2}|\log h|+\max _{2<j}\left\|u_{j}-u_{h_{j}}^{0}\right\|_{j}, \\
\rho\left\|u_{2}-u_{h_{2}}^{1}\right\|_{2} \leq \max _{2<j}\left\|u_{j}-u_{h_{j}}^{0}\right\|_{j}, \\
\left\|u_{1}-u_{h_{1}}^{1}\right\|_{1} \leq \max _{2<j}\left\|u_{j}-u_{h_{j}}^{0}\right\|_{j}
\end{gathered}
$$


By multiplying (227) by $\rho$ we get

$$
\begin{gathered}
\rho\left\|u_{2}-u_{h_{2}}^{1}\right\|_{2} \leq \rho C h^{2}|\log h|+\rho \max _{2<j}\left\|u_{j}-u_{h_{j}}^{0}\right\|_{j} \\
\rho\left\|u_{2}-u_{h_{2}}^{1}\right\|_{2} \leq \max _{2<j}\left\|u_{j}-u_{h_{j}}^{0}\right\|_{j}, \\
\left\|u_{1}-u_{h_{1}}^{1}\right\|_{1} \leq \max _{2<j}\left\|u_{j}-u_{h_{j}}^{0}\right\|_{j}
\end{gathered}
$$

We remark that $\rho\left\|u_{2}-u_{h_{2}}^{1}\right\|_{2}$ is bounded by both $\rho C h^{2}$ $|\log h|+\rho \max _{2<j}\left\|u_{j}-u_{h_{j}}^{0}\right\|_{j}$ and $\max _{2<j}\left\|u_{j}-u_{h_{j}}^{0}\right\|_{j}$ then

$$
\text { (c) } \max _{2<j}\left\|u_{j}-u_{h_{j}}^{0}\right\|_{j}<\rho C h^{2}|\log h|+\rho \max _{2<j}\left\|u_{j}-u_{h_{j}}^{0}\right\|_{j}
$$

or

(d) $\rho C h^{2}|\log h|+\rho \max _{2<j}\left\|u_{j}-u_{h_{j}}^{0}\right\|_{j}<\max _{2<j}\left\|u_{j}-u_{h_{j}}^{0}\right\|_{j}$.

That is

$$
\max _{2<j}\left\|u_{j}-u_{h_{j}}^{0}\right\|_{j}<\frac{\rho}{1-\rho} C h^{2}|\log h|
$$

or

$$
\frac{\rho}{1-\rho} C h^{2}|\log h|<\max _{2<j}\left\|u_{j}-u_{h_{j}}^{0}\right\|_{j}
$$

Which implies

$$
\max _{2<j}\left\|u_{j}-u_{h_{j}}^{0}\right\|_{j}<\frac{\rho}{1-\rho} C h^{2}|\log h|<C h^{2}|\log h|
$$

or

$$
\frac{\rho}{1-\rho} C h^{2}|\log h|<C h^{2}|\log h| \leq \max _{2<j}\left\|u_{j}-u_{h_{j}}^{0}\right\|_{j} .
$$

It is clear that only case $(\mathrm{d})$ is possible. By adding $C h^{2}|\log h|$ in (232) we get

$$
C h^{2}|\log h|+\frac{\rho}{1-\rho} C h^{2}|\log h|<C h^{2}|\log h|+\max _{2<j}\left\|u_{j}-u_{h_{j}}^{0}\right\|_{j}
$$

so

$$
\frac{1}{1-\rho} C h^{2}|\log h|<C h^{2}|\log h|+\max _{2<j}\left\|u_{j}-u_{h_{j}}^{0}\right\|_{j}
$$

comparing the last inequality with (227), we consider the two following possibilties

$$
\left\|u_{2}-u_{h_{2}}^{1}\right\|_{2}<\frac{1}{1-\rho} C h^{2}|\log h|
$$

or

$$
\frac{1}{1-\rho} C h^{2}|\log h|<\left\|u_{2}-u_{h_{2}}^{1}\right\|_{2}
$$

that is

$$
\left\|u_{2}-u_{h_{2}}^{1}\right\|_{2}<\frac{1}{1-\rho} C h^{2}|\log h|<C h^{2}|\log h|+\max _{2<j}\left\|u_{j}-u_{h_{j}}^{0}\right\|_{j}
$$

or

$$
\frac{1}{1-\rho} C h^{2}|\log h|<\left\|u_{2}-u_{h_{2}}^{1}\right\|_{2} \leq C h^{2}|\log h|+\max _{2<j}\left\|u_{j}-u_{h_{j}}^{0}\right\|_{j} .
$$

It is clear that the two possibilities are true so either there is a contradiction and case 3 is impossible or case 3 is possible only if

$$
\left\|u_{2}-u_{h_{2}}^{1}\right\|_{2}=\frac{1}{1-\rho} C h^{2}|\log h|
$$

So case 1 is impossible and in both cases 2 and 3 we obtain

$$
\left\|u_{2}-u_{h_{2}}^{1}\right\|_{2}=\frac{1}{1-\rho} C h^{2}|\log h| .
$$

Equations (208) and (242) imply

$$
\max _{1 \leq i<3}\left\|u_{i}-u_{h_{i}}^{1}\right\|_{i}=\frac{1}{1-\rho} C h^{2}|\log h| .
$$

We adopt the same approach for the other subdomains $\Omega_{i}$, $i=3, \ldots, m$ in order, we get

$$
\left\|u_{i}-u_{h_{i}}^{1}\right\|_{i}=\frac{1}{1-\rho} C h^{2}|\log h|, \quad i=1, \ldots, m .
$$

The remainder of the proof is by induction and is by adopting the same approach used in iteration one. The last step of the proof of this Part 2 is devoted to the subinterval $\rho \in[1 / 2,1[$ and is also by induction. Indeed for subdomain 1 and $n=0$, (73) implies

$$
\begin{aligned}
\left\|u_{1}-u_{h_{1}}^{1}\right\|_{1} \leq & C h^{2}|\log h| \\
& +\max \left\{\rho\left\|u_{1}-u_{h_{1}}^{1}\right\|_{1}, \max _{1<j}\left\|u_{j}-u_{h_{j}}^{0}\right\|_{j}\right\} .
\end{aligned}
$$

We have to distinguish the two following cases

$$
\text { (1) } \max \left\{\rho\left\|u_{1}-u_{h_{1}}^{1}\right\|_{1}, \max _{1<j}\left\|u_{j}-u_{h_{j}}^{0}\right\|_{j}\right\}=\rho\left\|u_{1}-u_{h_{1}}^{1}\right\|_{1}
$$

or

(2) $\max \left\{\rho\left\|u_{1}-u_{h_{1}}^{1}\right\|_{1}, \max _{1<j}\left\|u_{j}-u_{h_{j}}^{0}\right\|_{j}\right\}=\max _{1<j}\left\|u_{j}-u_{h_{j}}^{0}\right\|_{j}$.

Case 1 implies

$$
\begin{gathered}
\left\|u_{1}-u_{h_{1}}^{1}\right\|_{1} \leq C h^{2}|\log h|+\rho\left\|u_{1}-u_{h_{1}}^{1}\right\|_{1}, \\
\max _{1<j}\left\|u_{j}-u_{h_{j}}^{0}\right\|_{j} \leq \rho\left\|u_{1}-u_{h_{1}}^{1}\right\|_{1} .
\end{gathered}
$$


So

$$
\begin{aligned}
\left\|u_{1}-u_{h_{1}}^{1}\right\|_{1} & \leq \frac{1}{1-\rho} C h^{2}|\log h|, \\
C h^{2}|\log h| & <\max _{1<j}\left\|u_{j}-u_{h_{j}}^{0}\right\|_{j} \\
& \leq \rho\left\|u_{1}-u_{h_{1}}^{1}\right\|_{1} \\
& \leq \frac{\rho}{1-\rho} C h^{2}|\log h| .
\end{aligned}
$$

We remark that (249) coincides with (70). Case 2 implies

$$
\begin{gathered}
\left\|u_{1}-u_{h_{1}}^{1}\right\|_{1} \leq C h^{2}|\log h|+\max _{1<j}\left\|u_{j}-u_{h_{j}}^{0}\right\|_{j}, \\
\rho\left\|u_{1}-u_{h_{1}}^{1}\right\|_{1} \leq \max _{1<j}\left\|u_{j}-u_{h_{j}}^{0}\right\|_{j} \cdot
\end{gathered}
$$

By multiplying (250) by $\rho$ we get

$$
\begin{gathered}
\rho\left\|u_{1}-u_{h_{1}}^{1}\right\|_{1} \leq \rho C h^{2}|\log h|+\rho \max _{1<j}\left\|u_{j}-u_{h_{j}}^{0}\right\|_{j}, \\
\rho\left\|u_{1}-u_{h_{1}}^{1}\right\|_{1} \leq \max _{1<j}\left\|u_{j}-u_{h_{j}}^{0}\right\|_{j} .
\end{gathered}
$$

It is clear that $\rho\left\|u_{1}-u_{h_{1}}^{1}\right\|_{1}$ is bounded by both $\rho C h^{2}|\log h|+$ $\rho \max _{1<j}\left\|u_{j}-u_{h_{j}}^{0}\right\|_{j}$ and $\max _{1<j}\left\|u_{j}-u_{h_{j}}^{0}\right\|_{j}$, so

(a) $\max _{1<j}\left\|u_{j}-u_{h_{j}}^{0}\right\|_{j}<\rho C h^{2}|\log h|+\rho \max _{1<j}\left\|u_{j}-u_{h_{j}}^{0}\right\|_{j}$

or

(b) $\rho C h^{2}|\log h|+\rho \max _{1<j}\left\|u_{j}-u_{h_{j}}^{0}\right\|_{j}<\max _{1<j}\left\|u_{j}-u_{h_{j}}^{0}\right\|_{j}$.

Which implies

$$
\max _{1<j}\left\|u_{j}-u_{h_{j}}^{0}\right\|_{j}<\frac{\rho}{1-\rho} C h^{2}|\log h|
$$

or

$$
\frac{\rho}{1-\rho} C h^{2}|\log h|<\max _{1<j}\left\|u_{j}-u_{h_{j}}^{0}\right\|_{j}
$$

Then

$$
C h^{2}|\log h|<\max _{1<j}\left\|u_{j}-u_{h_{j}}^{0}\right\|_{j}<\frac{\rho}{1-\rho} C h^{2}|\log h|
$$

or

$$
C h^{2}|\log h| \leq \frac{\rho}{1-\rho} C h^{2}|\log h|<\max _{1<j}\left\|u_{j}-u_{h_{j}}^{0}\right\|_{j} .
$$

It is clear that both cases (a) and (b) are true. So either there is contradiction and case 2 is impossible or case 2 is possible and we must have

$$
\max _{1<j}\left\|u_{j}-u_{h_{j}}^{0}\right\|_{j}=\frac{\rho}{1-\rho} C h^{2}|\log h| .
$$

Then case 2 implies

$$
\begin{aligned}
\left\|u_{1}-u_{h_{1}}^{1}\right\|_{1} & \leq C h^{2}|\log h|+\max _{1<j}\left\|u_{j}-u_{h_{j}}^{0}\right\|_{j} \\
& =C h^{2}|\log h|+\frac{\rho}{1-\rho} C h^{2}|\log h| \\
& =\frac{1}{1-\rho} C h^{2}|\log h| .
\end{aligned}
$$

So in both cases 1 and 2 we get

$$
\left\|u_{1}-u_{h_{1}}^{1}\right\|_{1} \leq \frac{1}{1-\rho} C h^{2}|\log h|
$$

Similarly applying (73) to subdomain 2 we get in this situation (B)

$$
\begin{aligned}
&\left\|u_{2}-u_{h_{2}}^{1}\right\|_{2} \leq C h^{2}|\log h| \\
&+\max \left\{\rho\left\|u_{2}-u_{h_{2}}^{1}\right\|_{2},\right. \\
&\left.\left\|u_{1}-u_{h_{1}}^{1}\right\|_{1}, \max _{2<j}\left\|u_{j}-u_{h_{j}}^{0}\right\|_{j}\right\} .
\end{aligned}
$$

So we have to distinguish between the three following cases

$$
\begin{aligned}
& \text { (1) } \max \left\{\rho\left\|u_{2}-u_{h_{2}}^{1}\right\|_{2},\left\|u_{1}-u_{h_{1}}^{1}\right\|_{1}, \max _{2<j}\left\|u_{j}-u_{h_{j}}^{0}\right\|_{j}\right\} \\
& =\rho\left\|u_{2}-u_{h_{2}}^{1}\right\|_{2}
\end{aligned}
$$

or

(2) $\max \left\{\rho\left\|u_{2}-u_{h_{2}}^{1}\right\|_{2},\left\|u_{1}-u_{h_{1}}^{1}\right\|_{1}, \max _{2<j}\left\|u_{j}-u_{h_{j}}^{0}\right\|_{j}\right\}$

$$
=\left\|u_{1}-u_{h_{1}}^{1}\right\|_{1}
$$

or

$$
\begin{aligned}
& \text { (3) } \max \left\{\rho\left\|u_{2}-u_{h_{2}}^{1}\right\|_{2},\left\|u_{1}-u_{h_{1}}^{1}\right\|_{1}, \max _{2<j}\left\|u_{j}-u_{h_{j}}^{0}\right\|_{j}\right\} \\
& =\max _{2<j}\left\|u_{j}-u_{h_{j}}^{0}\right\|_{j} .
\end{aligned}
$$

Case 1 implies

$$
\begin{gathered}
\left\|u_{1}-u_{h_{2}}^{1}\right\|_{2} \leq C h^{2}|\log h|+\rho\left\|u_{2}-u_{h_{2}}^{1}\right\|_{2}, \\
\left\|u_{1}-u_{h_{1}}^{1}\right\|_{1} \leq \rho\left\|u_{2}-u_{h_{2}}^{1}\right\|_{2}, \\
\max _{2<j}\left\|u_{j}-u_{h_{j}}^{0}\right\|_{j} \leq \rho\left\|u_{2}-u_{h_{2}}^{1}\right\|_{2} .
\end{gathered}
$$


Then

$$
\begin{gathered}
\left\|u_{1}-u_{h_{2}}^{1}\right\|_{2} \leq \frac{1}{1-\rho} C h^{2}|\log h|, \\
\left\|u_{1}-u_{h_{1}}^{1}\right\|_{1} \leq \rho\left\|u_{2}-u_{h_{2}}^{1}\right\|_{2} \leq \frac{\rho}{1-\rho} C h^{2}|\log h| \\
\leq \frac{1}{1-\rho} C h^{2}|\log h|, \\
C h^{2}|\log h|<\max _{2<j}\left\|u_{j}-u_{h_{j}}^{0}\right\|_{j} \leq \rho\left\|u_{2}-u_{h_{2}}^{1}\right\|_{2} \\
\leq \frac{\rho}{1-\rho} C h^{2}|\log h| .
\end{gathered}
$$

Case 2 implies

$$
\begin{gathered}
\left\|u_{2}-u_{h_{2}}^{1}\right\|_{2} \leq C h^{2}|\log h|+\left\|u_{1}-u_{h_{1}}^{1}\right\|_{1}, \\
\rho\left\|u_{2}-u_{h_{2}}^{1}\right\|_{2} \leq\left\|u_{1}-u_{h_{1}}^{1}\right\|_{1}, \\
\max _{2<j}\left\|u_{j}-u_{h_{j}}^{0}\right\|_{j} \leq\left\|u_{1}-u_{h_{1}}^{1}\right\|_{1} .
\end{gathered}
$$

By multiplying (267) by $\rho$ we get

$$
\begin{gathered}
\rho\left\|u_{2}-u_{h_{2}}^{1}\right\|_{2} \leq \rho C h^{2}|\log h|+\rho\left\|u_{1}-u_{h_{1}}^{1}\right\|_{1}, \\
\rho\left\|u_{2}-u_{h_{2}}^{1}\right\|_{2} \leq\left\|u_{1}-u_{h_{1}}^{1}\right\|_{1}, \\
\max _{2<j}\left\|u_{j}-u_{h_{j}}^{0}\right\|_{j} \leq\left\|u_{1}-u_{h_{1}}^{1}\right\|_{1} .
\end{gathered}
$$

It is clear that $\rho\left\|u_{2}-u_{h_{2}}^{1}\right\|_{2}$ is bounded by both $\rho C h^{2}|\log h|+$ $\rho\left\|u_{1}-u_{h_{1}}^{1}\right\|_{1}$ and $\left\|u_{1}-u_{h_{1}}^{1}\right\|_{1}$ so
(a) $\left\|u_{1}-u_{h_{1}}^{1}\right\|_{1}<\rho C h^{2}|\log h|+\rho\left\|u_{1}-u_{h_{1}}^{1}\right\|_{1}$

or

(b) $\rho C h^{2}|\log h|+\rho\left\|u_{1}-u_{h_{1}}^{1}\right\|_{1}<\left\|u_{1}-u_{h_{1}}^{1}\right\|_{1}$.

That is

$$
\left\|u_{1}-u_{h_{1}}^{1}\right\|_{1}<\frac{\rho}{1-\rho} C h^{2}|\log h|
$$

or

$$
\frac{\rho}{1-\rho} C h^{2}|\log h|<\left\|u_{1}-u_{h_{1}}^{1}\right\|_{1} .
$$

Thus

$$
\left\|u_{1}-u_{h_{1}}^{1}\right\|_{1}<\frac{\rho}{1-\rho} C h^{2}|\log h|<\frac{1}{1-\rho} C h^{2}|\log h|
$$

or

$$
\frac{\rho}{1-\rho} C h^{2}|\log h|<\left\|u_{1}-u_{h_{1}}^{1}\right\|_{1} \leq \frac{1}{1-\rho} C h^{2}|\log h| .
$$

It is clear that both cases (a) and (b) are true because they both coincide with (208). So either there is contradiction and case 2 is impossible or case 2 is possible and we must have

$$
\left\|u_{1}-u_{h_{1}}^{1}\right\|_{1}=\frac{\rho}{1-\rho} C h^{2}|\log h| .
$$

With

$$
\begin{aligned}
C h^{2}|\log h| & <\max _{2<j}\left\|u_{j}-u_{h_{j}}^{0}\right\|_{j} \leq\left\|u_{1}-u_{h_{1}}^{1}\right\|_{1} \\
& =\frac{\rho}{1-\rho} C h^{2}|\log h|,
\end{aligned}
$$

So case 2 implies

$$
\begin{aligned}
\left\|u_{2}-u_{h_{2}}^{1}\right\|_{2} & \leq C h^{2}|\log h|+\left\|u_{1}-u_{h_{1}}^{1}\right\|_{1} \\
& =C h^{2}|\log h|+\frac{\rho}{1-\rho} C h^{2}|\log h|
\end{aligned}
$$

Then

$$
\left\|u_{2}-u_{h_{2}}^{1}\right\|_{2} \leq \frac{1}{1-\rho} C h^{2}|\log h| .
$$

Case 3 implies

$$
\begin{gathered}
\left\|u_{2}-u_{h_{2}}^{1}\right\|_{2} \leq C h^{2}|\log h|+\max _{2<j}\left\|u_{j}-u_{h_{j}}^{0}\right\|_{j}, \\
\rho\left\|u_{2}-u_{h_{2}}^{1}\right\|_{2} \leq \max _{2<j}\left\|u_{j}-u_{h_{j}}^{0}\right\|_{j}, \\
\left\|u_{1}-u_{h_{1}}^{1}\right\|_{1} \leq \max _{2<j}\left\|u_{j}-u_{h_{j}}^{0}\right\|_{j} .
\end{gathered}
$$

By multiplying (279) by $\rho$ we get

$$
\begin{gathered}
\rho\left\|u_{2}-u_{h_{2}}^{1}\right\|_{2} \leq \rho C h^{2}|\log h|+\rho \max _{2<j}\left\|u_{j}-u_{h_{j}}^{0}\right\|_{j}, \\
\rho\left\|u_{2}-u_{h_{2}}^{1}\right\|_{2} \leq \max _{2<j}\left\|u_{j}-u_{h_{j}}^{0}\right\|_{j} \\
\left\|u_{1}-u_{h_{1}}^{1}\right\|_{1} \leq \max _{2<j}\left\|u_{j}-u_{h_{j}}^{0}\right\|_{j} \cdot
\end{gathered}
$$

We remark that $\rho\left\|u_{2}-u_{h_{2}}^{1}\right\|_{2}$ is bounded by both $\rho C h^{2}|\log h|+\rho \max _{2<j}\left\|u_{j}-u_{h_{j}}^{0}\right\|_{j}$ and $\max _{2<j}\left\|u_{j}-u_{h_{j}}^{0}\right\|_{j}$ then

(c) $\max _{2<j}\left\|u_{j}-u_{h_{j}}^{0}\right\|_{j}<\rho C h^{2}|\log h|+\rho \max _{2<j}\left\|u_{j}-u_{h_{j}}^{0}\right\|_{j}$

or

(d) $\rho C h^{2}|\log h|+\rho \max _{2<j}\left\|u_{j}-u_{h_{j}}^{0}\right\|_{j}<\max _{2<j}\left\|u_{j}-u_{h_{j}}^{0}\right\|_{j}$.

That is

$$
\max _{2<j}\left\|u_{j}-u_{h_{j}}^{0}\right\|_{j}<\frac{\rho}{1-\rho} C h^{2}|\log h|
$$


or

$$
\frac{\rho}{1-\rho} C h^{2}|\log h|<\max _{2<j}\left\|u_{j}-u_{h_{j}}^{0}\right\|_{j}
$$

Which implies

$$
C h^{2}|\log h| \leq \max _{2<j}\left\|u_{j}-u_{h_{j}}^{0}\right\|_{j}<\frac{\rho}{1-\rho} C h^{2}|\log h|
$$

or

$$
C h^{2}|\log h| \leq \frac{\rho}{1-\rho} C h^{2}|\log h|<\max _{2<j}\left\|u_{j}-u_{h_{j}}^{0}\right\|_{j} .
$$

It is clear that both cases (c) and (d) are true. So either there is contradiction and case 3 is impossible or case 3 is possible only if.

$$
\max _{2<j}\left\|u_{j}-u_{h_{j}}^{0}\right\|_{j}=\frac{\rho}{1-\rho} C h^{2}|\log h|
$$

Then in case 3 we obtain

$$
\begin{aligned}
\left\|u_{2}-u_{h_{2}}^{1}\right\|_{2} & \leq C h^{2}|\log h|+\max _{2<j}\left\|u_{j}-u_{h_{j}}^{0}\right\|_{j} \\
& =C h^{2}|\log h|+\frac{\rho}{1-\rho} C h^{2}|\log h|
\end{aligned}
$$

Thus

$$
\left\|u_{2}-u_{h_{2}}^{1}\right\|_{2} \leq \frac{1}{1-\rho} C h^{2}|\log h| .
$$

With

$$
\left\|u_{1}-u_{h_{1}}^{1}\right\|_{1} \leq \max _{2<j}\left\|u_{j}-u_{h_{j}}^{0}\right\|_{j}=\frac{\rho}{1-\rho} C h^{2}|\log h| .
$$

We adopt the same approach for the other subdomains $\Omega_{i}$, $i=2, \ldots, m$ in order, we get

$$
\left\|u_{i}-u_{h_{i}}^{1}\right\|_{i} \leq \frac{1}{1-\rho} C h^{2}|\log h|, \quad i=1, \ldots, m .
$$

The remainder of the proof related to iterations $n \geq 2$ is by induction by adopting the same idea used in iteration one.

Part 3. We deal with situation (C). The proof related to this part 3 is also by induction and is so similar to the part 2 one. So we give it for the first subinterval $] 0,1 / 2[$. The one for the second subinterval $[1 / 2,1$ [ will be omitted. Indeed for $n=0$ and $i=1$ we use (73) in conjunction with (71) we get

$$
\begin{aligned}
\left\|u_{1}-u_{h_{1}}^{1}\right\|_{1} \leq & C h^{2}|\log h| \\
& +\max \left\{\rho\left\|u_{1}-u_{h_{1}}^{n+1}\right\|_{1}, \max _{1<j, j \in J}\left\|u_{j}-u_{h_{j}}^{n}\right\|_{j}\right\}
\end{aligned}
$$

We have to distinguish the two following cases

$$
\begin{gathered}
\text { (1) } \max \left\{\rho\left\|u_{1}-u_{h_{1}}^{1}\right\|_{1}, \max _{1<j, j \in J}\left\|u_{j}-u_{h_{j}}^{0}\right\|_{j}\right\} \\
=\rho\left\|u_{1}-u_{h_{1}}^{1}\right\|_{1}
\end{gathered}
$$

or

$$
\begin{gathered}
\text { (2) } \max \left\{\rho\left\|u_{1}-u_{h_{1}}^{1}\right\|_{1}, \max _{1<j, j \in J}\left\|u_{j}-u_{h_{j}}^{0}\right\|_{j}\right\} \\
=\max _{1<j, j \in J}\left\|u_{j}-u_{h_{j}}^{0}\right\|_{j} .
\end{gathered}
$$

Case 1 implies

$$
\begin{gathered}
\left\|u_{1}-u_{h_{1}}^{1}\right\|_{1} \leq \frac{1}{1-\rho} C h^{2}|\log h|, \\
\max _{1<j, j \in J}\left\|u_{j}-u_{h_{j}}^{0}\right\|_{j} \leq \rho\left\|u_{1}-u_{h_{1}}^{1}\right\|_{1} \\
\leq \frac{\rho}{1-\rho} C h^{2}|\log h|<C h^{2}|\log h| .
\end{gathered}
$$

We remark that (295) contradicts (71) so case 1 is impossible. Case 2 implies

$$
\begin{gathered}
\left\|u_{1}-u_{h_{1}}^{1}\right\|_{1} \leq C h^{2}|\log h|+\max _{1<j, j \in J}\left\|u_{j}-u_{h_{j}}^{0}\right\|_{j}, \\
\rho\left\|u_{1}-u_{h_{1}}^{1}\right\|_{1} \leq \max _{1<j, j \in J}\left\|u_{j}-u_{h_{j}}^{0}\right\|_{j} .
\end{gathered}
$$

By multiplying (296) by $\rho$ we get

$$
\begin{gathered}
\rho\left\|u_{1}-u_{h_{1}}^{1}\right\|_{1} \leq \rho C h^{2}|\log h|+\rho \max _{1<j, j \in J}\left\|u_{j}-u_{h_{j}}^{0}\right\|_{j}, \\
\rho\left\|u_{1}-u_{h_{1}}^{1}\right\|_{1} \leq \max _{1<j, j \in J}\left\|u_{j}-u_{h_{j}}^{0}\right\|_{j}
\end{gathered}
$$

It is clear that $\rho\left\|u_{1}-u_{h_{1}}^{1}\right\|_{1}$ is bounded by both $\rho C h^{2}|\log h|+$ $\rho \max _{1<j, j \in J}\left\|u_{j}-u_{h_{j}}^{0}\right\|_{j}$ and $\max _{1<j, j \in J}\left\|u_{j}-u_{h_{j}}^{0}\right\|_{j}$, so

$$
\begin{aligned}
& \text { (a) } \max _{1<j, j \in J}\left\|u_{j}-u_{h_{j}}^{0}\right\|_{j} \\
& \quad<\rho C h^{2}|\log h|+\rho \max _{1<j, j \in J}\left\|u_{j}-u_{h_{j}}^{0}\right\|_{j}
\end{aligned}
$$

or

$$
\begin{aligned}
& \text { (b) } \rho C h^{2}|\log h|+\rho \max _{1<j, j \in J}\left\|u_{j}-u_{h_{j}}^{0}\right\|_{j} \\
& <\max _{1<j, j \in J}\left\|u_{j}-u_{h_{j}}^{0}\right\|_{j} .
\end{aligned}
$$

Which implies

$$
\max _{1<j, j \in J}\left\|u_{j}-u_{h_{j}}^{0}\right\|_{j}<\frac{\rho}{1-\rho} C h^{2}|\log h|
$$

or

$$
\frac{\rho}{1-\rho} C h^{2}|\log h|<\max _{1<j, j \in J}\left\|u_{j}-u_{h_{j}}^{0}\right\|_{j}
$$

Then

$$
C h^{2}|\log h| \leq \max _{1<j, j \in J}\left\|u_{j}-u_{h_{j}}^{0}\right\|_{j}<\frac{\rho}{1-\rho} C h^{2}|\log h|
$$


or

$$
\frac{\rho}{1-\rho} C h^{2}|\log h|<C h^{2}|\log h| \leq \max _{1<j, j \in J}\left\|u_{j}-u_{h_{j}}^{0}\right\|_{j} .
$$

We can see that only case (b) is possible and by adding $C h^{2}|\log h|$ in (301) we get

$$
\begin{aligned}
& C h^{2}|\log h|+\frac{\rho}{1-\rho} C h^{2}|\log h| \\
& \quad<C h^{2}|\log h|+\max _{1<j, j \in J}\left\|u_{j}-u_{h_{j}}^{0}\right\|_{j}
\end{aligned}
$$

thus

$$
\frac{1}{1-\rho} C h^{2}|\log h|<C h^{2}|\log h|+\max _{1<j, j \in J}\left\|u_{j}-u_{h_{j}}^{0}\right\|_{j}
$$

comparing this last inequalty (305) with (296), we deduce the two following possibilities

$$
\left\|u_{1}-u_{h_{1}}^{1}\right\|_{1}<\frac{1}{1-\rho} C h^{2}|\log h|
$$

or

$$
\frac{1}{1-\rho} C h^{2}|\log h|<\left\|u_{1}-u_{h_{1}}^{1}\right\|_{1},
$$

which implies

$$
\begin{aligned}
\| u_{1}- & u_{h_{1}}^{1} \|_{1}<\frac{1}{1-\rho} C h^{2}|\log h| \\
& <C h^{2}|\log h|+\max _{1<j, j \in j}\left\|u_{j}-u_{h_{j}}^{0}\right\|_{j}
\end{aligned}
$$

or

$$
\begin{aligned}
& \frac{1}{1-\rho} C h^{2}|\log h|<\left\|u_{1}-u_{h_{1}}^{1}\right\|_{1} \\
& \quad \leq C h^{2}|\log h|+\max _{1<j, j \in J}\left\|u_{j}-u_{h_{j}}^{0}\right\|_{j} \cdot
\end{aligned}
$$

It is clear that the two possibilities are true so either there is a contradiction and case 2 is impossible or case 2 is possible only if

$$
\left\|u_{1}-u_{h_{1}}^{1}\right\|_{1}=\frac{1}{1-\rho} C h^{2}|\log h| .
$$

Similarly applying (73) to subdomain 2 in conjunction with (71) we get

$$
\begin{aligned}
&\left\|u_{2}-u_{h_{2}}^{1}\right\|_{2} \leq C h^{2}|\log h| \\
&+\max \left\{\rho\left\|u_{2}-u_{h_{2}}^{1}\right\|_{2},\left\|u_{1}-u_{h_{1}}^{1}\right\|_{1},\right. \\
&\left.\max _{2<j, j \in j}\left\|u_{j}-u_{h_{j}}^{0}\right\|_{j}\right\} .
\end{aligned}
$$

So we have to distinguish between the three following cases

$$
\begin{aligned}
& \text { (1) } \max \left\{\rho\left\|u_{2}-u_{h_{2}}^{1}\right\|_{2},\left\|u_{1}-u_{h_{1}}^{1}\right\|_{1}, \max _{2<j, j \in j}\left\|u_{j}-u_{h_{j}}^{0}\right\|_{j}\right\} \\
& =\rho\left\|u_{2}-u_{h_{2}}^{1}\right\|_{2}
\end{aligned}
$$

or

$$
\begin{aligned}
& \text { (2) } \max \left\{\rho\left\|u_{2}-u_{h_{2}}^{1}\right\|_{2},\left\|u_{1}-u_{h_{1}}^{1}\right\|_{1}, \max _{2<j, j \in J}\left\|u_{j}-u_{h_{j}}^{0}\right\|_{j}\right\} \\
& =\left\|u_{1}-u_{h_{1}}^{1}\right\|_{1}
\end{aligned}
$$

or

$$
\begin{aligned}
& \text { (3) } \max \left\{\rho\left\|u_{2}-u_{h_{2}}^{1}\right\|_{2},\left\|u_{1}-u_{h_{1}}^{1}\right\|_{1}, \max _{2<j, j \in J}\left\|u_{j}-u_{h_{j}}^{0}\right\|_{j}\right\} \\
& =\max _{2<j, j \in J}\left\|u_{j}-u_{h_{j}}^{0}\right\|_{j} .
\end{aligned}
$$

Case 1 implies

$$
\begin{gathered}
\left\|u_{2}-u_{h_{2}}^{1}\right\|_{2} \leq C h^{2}|\log h|+\rho\left\|u_{2}-u_{h_{2}}^{1}\right\|_{2}, \\
\left\|u_{1}-u_{h_{1}}^{1}\right\|_{1} \leq \rho\left\|u_{2}-u_{h_{2}}^{1}\right\|_{2}, \\
\max _{2<j, j \in j}\left\|u_{j}-u_{h_{j}}^{0}\right\|_{j} \leq \rho\left\|u_{2}-u_{h_{2}}^{1}\right\|_{2} .
\end{gathered}
$$

Then

$$
\begin{gathered}
\left\|u_{2}-u_{h_{2}}^{1}\right\|_{2} \leq \frac{1}{1-\rho} C h^{2}|\log h|, \\
\frac{1}{1-\rho} C h^{2}|\log h|=\left\|u_{1}-u_{h_{1}}^{1}\right\|_{1} \\
\leq \rho\left\|u_{2}-u_{h_{2}}^{1}\right\|_{2} \leq \frac{\rho}{1-\rho} C h^{2}|\log h|, \\
\max _{2<j, j \in j}\left\|u_{j}-u_{h_{j}}^{0}\right\|_{j} \leq \rho\left\|u_{2}-u_{h_{2}}^{1}\right\|_{2} \leq \frac{\rho}{1-\rho} C h^{2}|\log h| \\
<C h^{2}|\log h| .
\end{gathered}
$$

Equation (316) contradicts (71) so case 1 is impossible. Case 2 implies

$$
\begin{gathered}
\left\|u_{2}-u_{h_{2}}^{1}\right\|_{2} \leq C h^{2}|\log h|+\left\|u_{1}-u_{h_{1}}^{1}\right\|_{1}, \\
\rho\left\|u_{2}-u_{h_{2}}^{1}\right\|_{2} \leq\left\|u_{1}-u_{h_{1}}^{1}\right\|_{1}, \\
\max _{2<j, j \in J}\left\|u_{j}-u_{h_{j}}^{0}\right\|_{j} \leq\left\|u_{1}-u_{h_{1}}^{1}\right\|_{1} .
\end{gathered}
$$

By multiplying (317) by $\rho$ we get

$$
\begin{gathered}
\rho\left\|u_{2}-u_{h_{2}}^{1}\right\|_{2} \leq \rho C h^{2}|\log h|+\rho\left\|u_{1}-u_{h_{1}}^{1}\right\|_{1}, \\
\rho\left\|u_{2}-u_{h_{2}}^{1}\right\|_{2} \leq\left\|u_{1}-u_{h_{1}}^{1}\right\|_{1}, \\
\max _{2<j, j \in J}\left\|u_{j}-u_{h_{j}}^{0}\right\|_{j} \leq\left\|u_{1}-u_{h_{1}}^{1}\right\|_{1} .
\end{gathered}
$$


It is clear that $\rho\left\|u_{2}-u_{h_{2}}^{1}\right\|_{2}$ is bounded by both $\rho C h^{2}|\log h|+$ $\rho\left\|u_{1}-u_{h_{1}}^{1}\right\|_{1}$ and $\left\|u_{1}-u_{h_{1}}^{1}\right\|_{1}$ so

(a) $\left\|u_{1}-u_{h_{1}}^{1}\right\|_{1}<\rho C h^{2}|\log h|+\rho\left\|u_{1}-u_{h_{1}}^{1}\right\|_{1}$

or

(b) $\rho C h^{2}|\log h|+\rho\left\|u_{1}-u_{h_{1}}^{1}\right\|_{1}<\left\|u_{1}-u_{h_{1}}^{1}\right\|_{1}$.

That is

$$
\left\|u_{1}-u_{h_{1}}^{1}\right\|_{1}<\frac{\rho}{1-\rho} C h^{2}|\log h|
$$

or

$$
\frac{\rho}{1-\rho} C h^{2}|\log h|<\left\|u_{1}-u_{h_{1}}^{1}\right\|_{1} .
$$

It is clear that only case (b) is true because it coincides with (310) and by adding $C h^{2}|\log h|$ in (322) we get

$$
\begin{aligned}
\frac{1}{1-\rho} C h^{2}|\log h| & =C h^{2}|\log h|+\frac{\rho}{1-\rho} C h^{2}|\log h| \\
& <C h^{2}|\log h|+\left\|u_{1}-u_{h_{1}}^{1}\right\|_{1}
\end{aligned}
$$

comparing this last inequalty with (317), we distingush the two following possibilities

$$
\left\|u_{2}-u_{h_{2}}^{1}\right\|_{2}<\frac{1}{1-\rho} C h^{2}|\log h|
$$

or

$$
\frac{1}{1-\rho} C h^{2}|\log h|<\left\|u_{2}-u_{h_{2}}^{1}\right\|_{2}
$$

which imply

$$
\left\|u_{2}-u_{h_{2}}^{1}\right\|_{2}<\frac{1}{1-\rho} C h^{2}|\log h|<C h^{2}|\log h|+\left\|u_{1}-u_{h_{1}}^{1}\right\|_{1}
$$

or

$$
\frac{1}{1-\rho} C h^{2}|\log h|<\left\|u_{2}-u_{h_{2}}^{1}\right\|_{2} \leq C h^{2}|\log h|+\left\|u_{1}-u_{h_{1}}^{1}\right\|_{1} .
$$

It is clear that the two possibilities are true so either there is a contradiction and case 2 is impossible or case 2 is possible only if

$$
\left\|u_{2}-u_{h_{2}}^{1}\right\|_{2}=\frac{1}{1-\rho} C h^{2}|\log h| .
$$

Case 3 implies

$$
\begin{gathered}
\left\|u_{2}-u_{h_{2}}^{1}\right\|_{2} \leq C h^{2}|\log h|+\max _{2<j, j \in J}\left\|u_{j}-u_{h_{j}}^{0}\right\|_{j}, \\
\rho\left\|u_{2}-u_{h_{2}}^{1}\right\|_{2} \leq \max _{2<j, j \in J}\left\|u_{j}-u_{h_{j}}^{0}\right\|_{j}, \\
\left\|u_{1}-u_{h_{1}}^{1}\right\|_{1} \leq \max _{2<j, j \in J}\left\|u_{j}-u_{h_{j}}^{0}\right\|_{j} .
\end{gathered}
$$

By multiplying (329) by $\rho$ we get

$$
\begin{gathered}
\rho\left\|u_{2}-u_{h_{2}}^{1}\right\|_{2} \leq \rho C h^{2}|\log h|+\rho \max _{2<j, j \in J}\left\|u_{j}-u_{h_{j}}^{0}\right\|_{j}, \\
\rho\left\|u_{2}-u_{h_{2}}^{1}\right\|_{2} \leq \max _{2<j, j \in J}\left\|u_{j}-u_{h_{j}}^{0}\right\|_{j}, \\
\left\|u_{1}-u_{h_{1}}^{1}\right\|_{1} \leq \max _{2<j, j \in J}\left\|u_{j}-u_{h_{j}}^{0}\right\|_{j} .
\end{gathered}
$$

We remark that $\rho\left\|u_{2}-u_{h_{2}}^{1}\right\|_{2}$ is bounded by both $\rho C h^{2}|\log h|+$ $\rho \max _{2<j, j \in J}\left\|u_{j}-u_{h_{j}}^{0}\right\|_{j}$ and $\max _{2<j, j \in J}\left\|u_{j}-u_{h_{j}}^{0}\right\|_{j}$ then

$$
\begin{aligned}
& \text { (c) } \max _{2<j, j \in J}\left\|u_{j}-u_{h_{j}}^{0}\right\|_{j} \\
& \quad<\rho C h^{2}|\log h|+\rho \max _{2<j, j \in J}\left\|u_{j}-u_{h_{j}}^{0}\right\|_{j}
\end{aligned}
$$

or

$$
\begin{aligned}
& \text { (d) } \rho C h^{2}|\log h|+\rho_{2<j, j \in J} \max \left\|u_{j}-u_{h_{j}}^{0}\right\|_{j} \\
& <\max _{2<j, j \in J}\left\|u_{j}-u_{h_{j}}^{0}\right\|_{j}
\end{aligned}
$$

That is

$$
\max _{2<j, j \in J}\left\|u_{j}-u_{h_{j}}^{0}\right\|_{j}<\frac{\rho}{1-\rho} C h^{2}|\log h|
$$

or

$$
\frac{\rho}{1-\rho} C h^{2}|\log h|<\max _{2<j, j \in J}\left\|u_{j}-u_{h_{j}}^{0}\right\|_{j} .
$$

Which implies

$$
\max _{2<j, j \in J}\left\|u_{j}-u_{h_{j}}^{0}\right\|_{j}<\frac{\rho}{1-\rho} C h^{2}|\log h|<C h^{2}|\log h|
$$

or

$$
\frac{\rho}{1-\rho} C h^{2}|\log h|<C h^{2}|\log h| \leq \max _{2<j, j \in J}\left\|u_{j}-u_{h_{j}}^{0}\right\|_{j} .
$$

It is clear that only case (d) is possible. By adding $C h^{2}|\log h|$ in (334) we get

$$
\begin{aligned}
& C h^{2}|\log h|+\frac{\rho}{1-\rho} C h^{2}|\log h| \\
& <C h^{2}|\log h|+\max _{2<j, j \in j}\left\|u_{j}-u_{h_{j}}^{0}\right\|_{j}
\end{aligned}
$$

so

$$
\frac{1}{1-\rho} C h^{2}|\log h|<C h^{2}|\log h|+\max _{2<j, j \in J}\left\|u_{j}-u_{h_{j}}^{0}\right\|_{j}
$$


comparing the last inequality with (329), we consider the two following possibilties

$$
\left\|u_{2}-u_{h_{2}}^{1}\right\|_{2}<\frac{1}{1-\rho} C h^{2}|\log h|
$$

or

$$
\frac{1}{1-\rho} C h^{2}|\log h|<\left\|u_{2}-u_{h_{2}}^{1}\right\|_{2}
$$

that is

$$
\begin{aligned}
\| u_{2}- & u_{h_{2}}^{1} \|_{2}<\frac{1}{1-\rho} C h^{2}|\log h| \\
& <C h^{2}|\log h|+\max _{2<j, j \in J}\left\|u_{j}-u_{h_{j}}^{0}\right\|_{j}
\end{aligned}
$$

or

$$
\begin{aligned}
& \frac{1}{1-\rho} C h^{2}|\log h|<\left\|u_{2}-u_{h_{2}}^{1}\right\|_{2} \\
& \leq C h^{2}|\log h|+\max _{2<j, j \in J}\left\|u_{j}-u_{h_{j}}^{0}\right\|_{j}
\end{aligned}
$$

It is clear that the two possibilities are true so either there is a contradiction and case 3 is impossible or case 3 is possible only if

$$
\left\|u_{2}-u_{h_{2}}^{1}\right\|_{2}=\frac{1}{1-\rho} C h^{2}|\log h| .
$$

So case 1 is impossible and in both cases 2 and 3 we obtain

$$
\left\|u_{2}-u_{h_{2}}^{1}\right\|_{2}=\frac{1}{1-\rho} C h^{2}|\log h|
$$

Equations (310) and (344) imply

$$
\max _{1 \leq i<3}\left\|u_{i}-u_{h_{i}}^{1}\right\|_{i}=\frac{1}{1-\rho} C h^{2}|\log h| .
$$

We adopt the same approach for the other subdomains $\Omega_{i}$, $i=3, \ldots, m$ in order, we get

$$
\left\|u_{i}-u_{h_{i}}^{1}\right\|_{i}=\frac{1}{1-\rho} C h^{2}|\log h|, \quad i=1, \ldots, m .
$$

The remainder of the proof is by induction and is by adopting the same approach used in iteration one.

\section{Conflict of Interests}

The author declares that there is no conflict of interests regarding the publication of this paper.

\section{References}

[1] P. L. Lions, "On the Schwarz alternating method. I," in Proceedings of the 1st International Symposium on Domain Decomposition Methods for Partial Differential Equations, pp. 1-42, SIAM, 1988.
[2] P. L. Lions, "On the Schwarz alternating method. II. Stochastic interpretation and order properties," in Proceedings of the 2nd International Symposium on Domain Decomposition Methods for Partial Differential Equations, pp. 47-70, SIAM, 1989.

[3] S.-H. Lui, "On monotone and Schwarz alternating methods for nonlinear elliptic PDES," Mathematical Modelling and Numerical Analysis, vol. 35, no. 1, pp. 1-15, 2001.

[4] S. H. Lui, "On linear monotone iteration and Schwarz methods for nonlinear elliptic PDES," Numerische Mathematik, vol. 93, no. 1, pp. 109-129, 2002.

[5] R. Glowinski, G. H. Golub, G. A. Meurant, and J. Periaux, Eds., Proceedings of the First International Symposium on Domain Decomposition Methods for Partial Differential Equations, SIAM, Philadelphia, PA, USA, 1988.

[6] A. Harbi and M. Boulbrachene, "Maximum norm analysis of a nonmatching grids method for nonlinear elliptic PDES," Journal of Applied Mathematics, vol. 2011, Article ID 605140, 18 pages, 2011.

[7] A. Harbi, "Maximum norm analysis of a nonmatching grids method for nonlinear elliptic boundary value problem $-\Delta u=$ $f(u)$," in Proceeding of the International Conference on Applied Mathematics and Numerical Analysis, 2013, WASET issue 78.

[8] M. Boulbrachene, Ph. Cortey-Dumont, and J. C. Miellou, "Mixing finite elements and finite differences in a subdomain method," in Domain Decomposition for Partial Differential Equations, pp. 198-216, SIAM, Philadelphia, PA, USA, 1988.

[9] X.-C. Cai, T. P. Mathew, and M. V. Sarkis, "Maximum norm analysis of overlapping nonmatching grid discretizations of elliptic equations," SIAM Journal on Numerical Analysis, vol. 37, no. 5, pp. 1709-1728, 2000.

[10] T. P. Mathew and G. Russo, "Maximum norm stability of difference schemes for parabolic equations on overset nonmatching space-time grids," Mathematics of Computation, vol. 72, no. 242, pp. 619-656, 2003.

[11] J. Nitsche, " $L^{\infty}$-convergence of finite element approximations," in Proceedings of the Symposium on Mathematical Aspects of Finite Element Methods, vol. 606 of Lecture Notes in Mathematics, pp. 261-274, 1977.

[12] C. V. Pao, Nonlinear Parabolic and Elliptic Equations, Plenum Press, New York, NY, USA, 1992.

[13] L. Badea, "A generalization of the Schwarz alternating method to an arbitrary number of subdomains," Numerische Mathematik, vol. 55, no. 1, pp. 61-81, 1989.

[14] J. Karátson and S. Korotov, "Discrete maximum principles for finite element solutions of nonlinear elliptic problems with mixed boundary conditions," Numerische Mathematik, vol. 99, no. 4, pp. 669-698, 2005.

[15] P. G. Ciarlet and P.-A. Raviart, "Maximum principle and uniform convergence for the finite element method," Computer Methods in Applied Mechanics and Engineering, vol. 2, no. 1, pp. $17-31,1973$. 


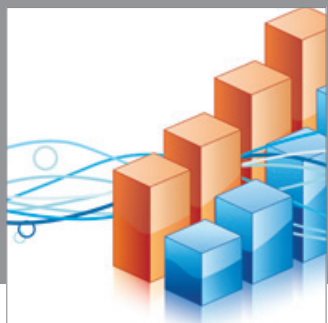

Advances in

Operations Research

mansans

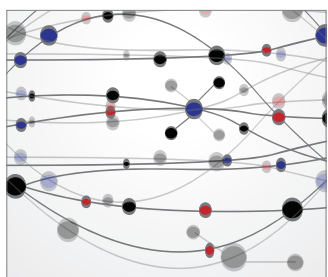

The Scientific World Journal
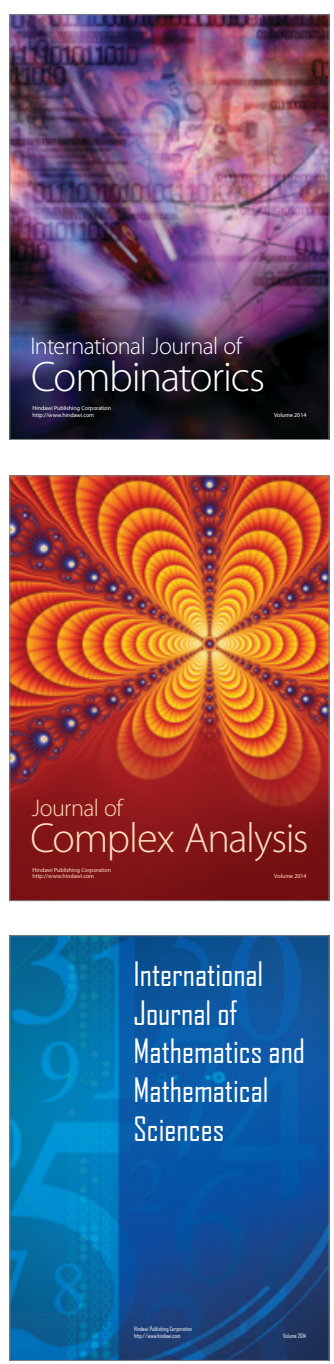
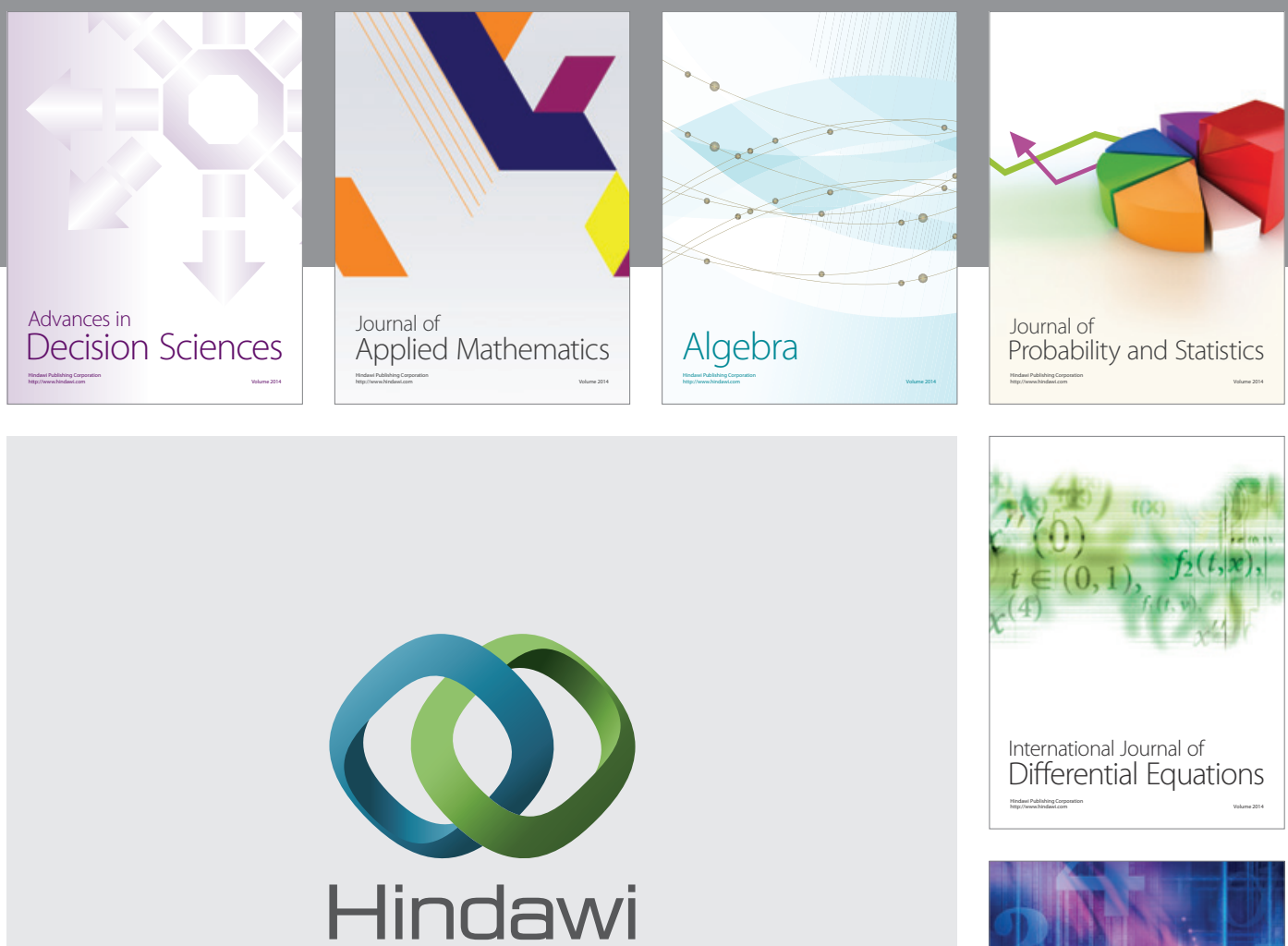

Submit your manuscripts at http://www.hindawi.com
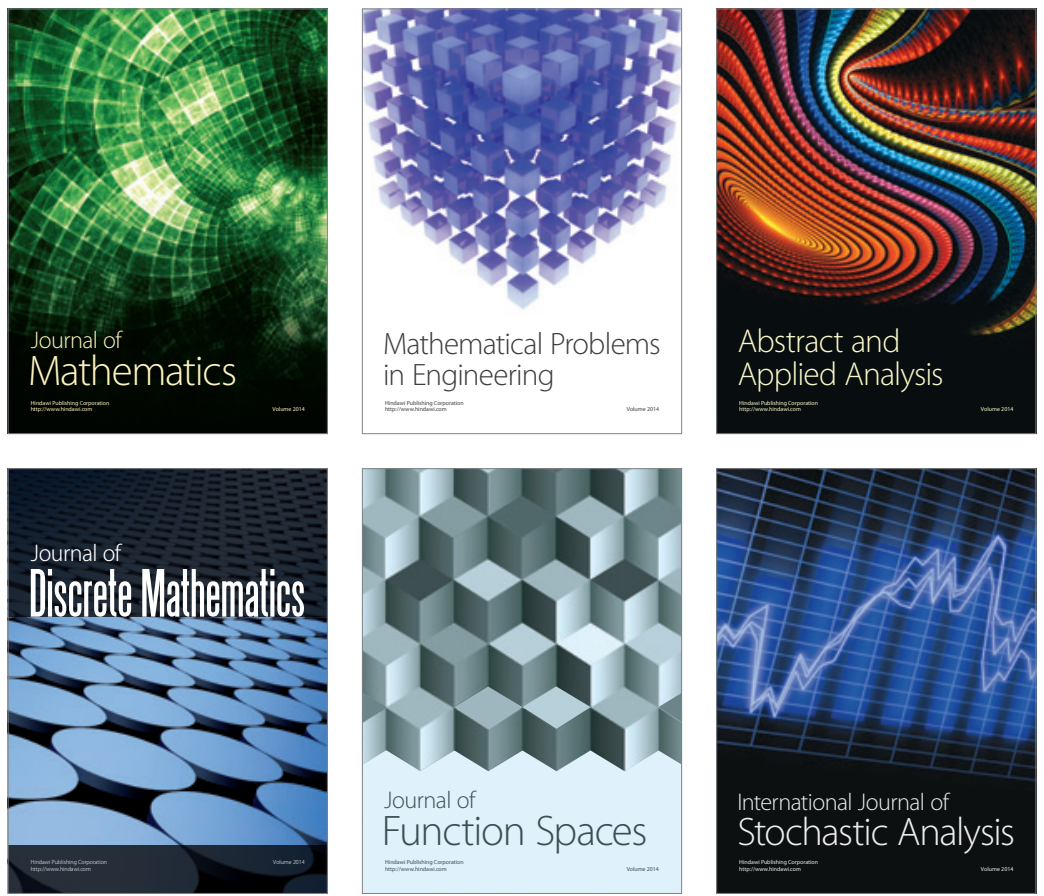

Journal of

Function Spaces

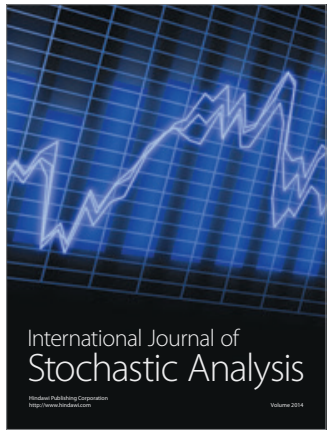

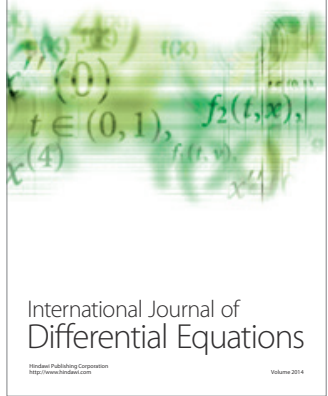
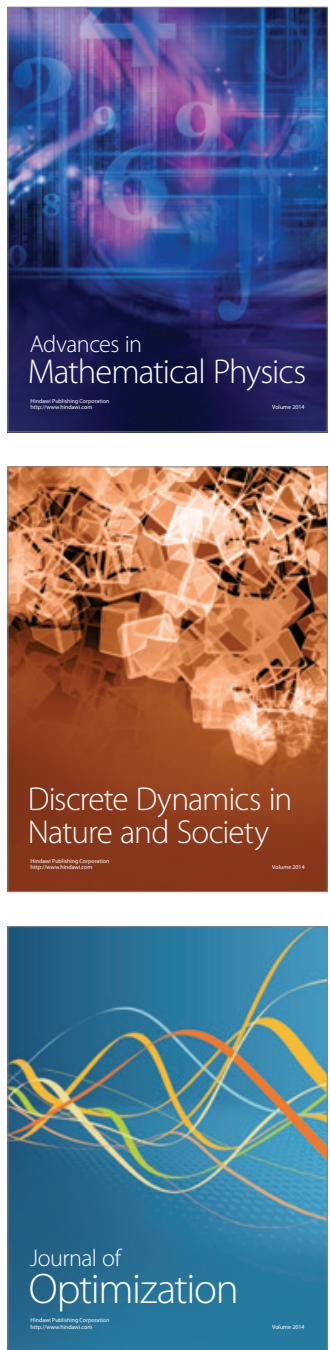